\title{
On the Kunen-Shelah properties in Banach spaces
}

\author{
by \\ Antonio S. Granero (Madrid), Mar Jiménez (Madrid), \\ Alejandro Montesinos (Madrid), José P. Moreno (Madrid) and \\ AnatoliJ PlichKo (Kirovograd)
}

\begin{abstract}
We introduce and study the Kunen-Shelah properties $\mathrm{KS}_{i}, i=0,1, \ldots, 7$. Let us highlight some of our results for a Banach space $X$ : (1) $X^{*}$ has a $w^{*}$-nonseparable equivalent dual ball iff $X$ has an $\omega_{1}$-polyhedron (i.e., a bounded family $\left\{x_{i}\right\}_{i<\omega_{1}}$ such that $x_{j} \notin \overline{\mathrm{co}}\left(\left\{x_{i}: i \in \omega_{1} \backslash\{j\}\right\}\right)$ for every $\left.j \in \omega_{1}\right)$ iff $X$ has an uncountable bounded almost biorthogonal system (UBABS) of type $\eta$ for some $\eta \in[0,1)$ (i.e., a bounded family $\left\{\left(x_{\alpha}, f_{\alpha}\right)\right\}_{1 \leq \alpha<\omega_{1}} \subset X \times X^{*}$ such that $f_{\alpha}\left(x_{\alpha}\right)=1$ and $\left|f_{\alpha}\left(x_{\beta}\right)\right| \leq \eta$ if $\left.\alpha \neq \beta\right)$; (2) if $X$ has an uncountable $\omega$-independent system then $X$ has an UBABS of type $\eta$ for every $\eta \in(0,1)$; (3) if $X$ does not have the property (C) of Corson, then $X$ has an $\omega_{1}$-polyhedron; (4) $X$ has no $\omega_{1}$-polyhedron iff $X$ has no convex right-separated $\omega_{1}$-family (i.e., a bounded family $\left\{x_{i}\right\}_{i<\omega_{1}}$ such that $x_{j} \notin \overline{\mathrm{co}}\left(\left\{x_{i}: j<i<\omega_{1}\right\}\right)$ for every $\left.j \in \omega_{1}\right)$ iff every $w^{*}$-closed convex subset of $X^{*}$ is $w^{*}$-separable iff every convex subset of $X^{*}$ is $w^{*}$-separable iff $\mu(X)=1$, $\mu(X)$ being the Finet-Godefroy index of $X$ (see [1]).
\end{abstract}

1. Introduction. If $X$ is a Banach space and $\theta$ an ordinal, a family $\left\{x_{\alpha}: \alpha<\theta\right\} \subset X$ is said to be a $\theta$-basic sequence if there exists $1 \leq K<\infty$ such that for every $n<m$ in $\mathbb{N}$, any $\lambda_{i} \in \mathbb{R}, i=1, \ldots, m$, and $\alpha_{1}<\ldots<$ $\alpha_{m}<\theta$ we have $\left\|\sum_{i=1}^{n} \lambda_{i} x_{\alpha_{i}}\right\| \leq K\left\|\sum_{i=1}^{m} \lambda_{i} x_{\alpha_{i}}\right\|$. A family $\left\{x_{i}\right\}_{i \in I} \subset X$ is a basic sequence if it is a $\theta$-basic sequence for some ordinal $\theta$. If $K=1$ the basic sequence is said to be monotone. A biorthogonal system in $X$ is a family $\left\{\left(x_{i}, x_{i}^{*}\right): i \in I\right\} \subset X \times X^{*}$ such that $x_{i}^{*}\left(x_{i}\right)=1$ and $x_{i}^{*}\left(x_{j}\right)=0$ for $i, j \in I, i \neq j$. A Markushevich system (for short, an M-system) in $X$ is a biorthogonal system $\left\{\left(x_{i}, x_{i}^{*}\right): i \in I\right\}$ in $X$ such that $\left\{x_{i}^{*}: i \in I\right\}$ is total on $\left[\overline{\left.\left\{x_{i}: i \in I\right\}\right]}\right.$ (see [14]).

It is well known (see [14, p. 599]) that if the density of a Banach space $X$ satisfies $\operatorname{Dens}(X) \geq \aleph_{1}$, then $X$ has a monotone $\omega_{1}$-basic sequence. Also if $\operatorname{Dens}(X)>\mathfrak{c}$, then $X$ has a monotone $\omega_{1}$-basic sequence, because in

2000 Mathematics Subject Classification: 46B20, 46B26.

Key words and phrases: uncountable basic sequences, biorthogonal and Markushevich systems, $\omega$-independence, Kunen-Shelah properties.

Supported in part by the DGICYT grant PB97-0240 (A. S. Granero and A. Montesinos) and grant PB97-0377 (M. Jiménez and J. P. Moreno). 
this case an easy calculation shows that $w^{*}$-Dens $\left(X^{*}\right) \geq \aleph_{1}$. However, if $\aleph_{1} \leq \operatorname{Dens}(X) \leq \mathfrak{c}$ and $w^{*}$-Dens $\left(X^{*}\right) \leq \aleph_{0}$, then $X$ can fail to have an uncountable basic sequence, even an uncountable biorthogonal system. Indeed, under the axiom $\diamond_{\aleph_{1}}$ (which implies the continuum hypothesis $(\mathrm{CH})$ ), Shelah [13] constructed a nonseparable Banach space $S$ that fails to have an uncountable biorthogonal system. Later Kunen [8, p. 1123] constructed under $(\mathrm{CH})$ a Hausdorff compact space $K$ such that $C(K)$ is nonseparable and has no uncountable biorthogonal system, among other interesting pathological properties.

A Banach space $X$ is said to have the Kunen-Shelah property $\mathrm{KS}_{0}$ (resp. $\mathrm{KS}_{1}$ ) if $X$ has no uncountable basic sequence (resp. uncountable Markushevich system). A Banach space $X$ is said to have the KunenShelah property $\mathrm{KS}_{2}$ if $X$ has no uncountable biorthogonal system. Clearly, $\mathrm{KS}_{2} \Rightarrow \mathrm{KS}_{1} \Rightarrow \mathrm{KS}_{0}$.

The first example of a Banach space $X$ such that $X \in \mathrm{KS}_{0}$ but $X \notin \mathrm{KS}_{2}$ was given in [9]; it is the space of Johnson-Lindenstrauss $\mathrm{JL}_{2}$ (see [5]). The properties $\mathrm{KS}_{2}$ and $\mathrm{KS}_{1}$ were separated in [2] (see also [1]), where it was proved that if a Banach space $X$ has the property (C) of Corson and $w^{*}$-Dens $\left(X^{*}\right) \leq \aleph_{0}$, then $X \in \mathrm{KS}_{1}$.

Question 1. Does there exist a Banach space $X$ such that $X \in \mathrm{KS}_{0}$ but $X \notin \mathrm{KS}_{1}$ ?

In this paper we study some structures similar to uncountable biorthogonal systems, namely: uncountable $\omega$-independent families, $\omega_{1}$-polyhedrons, uncountable bounded almost biorthogonal systems (UBABS), etc. The lack of these structures defines the Kunen-Shelah properties $\mathrm{KS}_{3}, \mathrm{KS}_{4}$, etc.

In Section 2 we prove that a Banach space $X$ has an $\omega_{1}$-polyhedron iff $X$ has an UBABS iff $X^{*}$ has a $w^{*}$-nonseparable dual equivalent ball. Section 3 deals with uncountable $\omega$-independent families. In Section 4 it is proved that $X$ has no $\omega_{1}$-polyhedron iff every $w^{*}$-closed convex subset of $X^{*}$ is $w^{*}$-separable. In Section 5 we answer some questions posed by Finet and Godefroy [1] concerning the index $\mu(X)$. In Section 6 we prove that a space $X$ has no convex right-separated $\omega_{1}$-family iff every $w^{*}$-closed convex subset of $X^{*}$ is $w^{*}$-separable. Finally, in Section 7 we show that $X$ has an $\omega_{1}$-polyhedron iff $X$ has a convex right-separated $\omega_{1}$-family, whence every $w^{*}$-closed convex subset of $X^{*}$ is $w^{*}$-separable iff every convex subset of $X^{*}$ is so.

Let us introduce some notation. $\omega_{1}$ is the first uncountable ordinal, $|A|$ the cardinality of the set $A$, and $\mathfrak{c}=|\mathbb{R}|$. If $X$ is a Banach space, $X^{*}$ denotes its dual, $B(X)$ and $S(X)$ the closed unit ball and sphere of $X$, resp., and $B(x, r)$ the closed ball with radius $r$ and center $x$. If $A \subset X$ we denote by $[A]$ the linear subspace spanned by $A$. Recall that a Banach space $X$ is 
said to have the property (C) of Corson (for short, $X \in(\mathrm{C})$ ) if $\bigcap_{i \in I} C_{i} \neq \emptyset$ whenever $\left\{C_{i}: i \in I\right\}$ is a family of closed bounded convex subsets of $X$ with the countable intersection property, i.e., $\emptyset \neq \bigcap_{i \in J} C_{i}$ for every countable subset $J \subset I$.

2. UBABS and $\omega_{1}$-polyhedrons. If $X$ is a Banach space, a bounded family $\left\{\left(x_{\alpha}, f_{\alpha}\right)\right\}_{1 \leq \alpha<\omega_{1}} \subset X \times X^{*}$ is said to be an uncountable bounded almost biorthogonal system (for short, an UBABS) if there exists a real number $0 \leq \eta<1$ such that $f_{\alpha}\left(x_{\alpha}\right)=1$ and $f_{\alpha}\left(x_{\beta}\right) \leq \eta$ if $\alpha \neq \beta$. If in addition $\left|f_{\alpha}\left(x_{\beta}\right)\right| \leq \eta$ for $\alpha \neq \beta$, then the UBABS is said to be of type $\eta$. Define the index $\tau(X)$ as follows:

$$
\tau(X)=\inf \{0 \leq \eta<1: X \text { has an UBABS of type } \eta\}
$$

where $\inf \{\emptyset\}=1$. Clearly, $\tau(X)$ is invariant under isomorphisms and: (1) if $X$ has an uncountable biorthogonal system, then $\tau(X)=0 ;(2) \tau(X)<1$ iff $X$ has an UBABS.

If $\tau$ is a cardinal, a bounded family $\left\{x_{i}\right\}_{i \in \tau}$ in a Banach space $X$ is said to be a $\tau$-polyhedron iff $x_{j} \notin \overline{\mathrm{co}}\left(\left\{x_{i}\right\}_{i \in \tau \backslash\{j\}}\right)$ for every $j \in \tau$. In a dual Banach space $X^{*}$ one can define a $w^{*}-\tau$-polyhedron in an analogous way, using the $w^{*}$-topology instead of the w-topology.

Proposition 2.1. A Banach space $X$ has an $\omega_{1}$-polyhedron iff $X^{*}$ has a $w^{*}-\omega_{1}$-polyhedron.

Proof. Let $\left\{x_{\alpha}\right\}_{\alpha<\omega_{1}} \subset B(X)$ be an $\omega_{1}$-polyhedron. By the Hahn-Banach Theorem there exists $f_{\alpha} \in S\left(X^{*}\right)$ such that

$$
f_{\alpha}\left(x_{\alpha}\right)>\sup \left\{f_{\alpha}\left(x_{i}\right): i \in \omega_{1} \backslash\{\alpha\}\right\}=: e_{\alpha} .
$$

By passing to a subsequence, we can suppose that there exist $0<\varepsilon<\infty$ and $r \in \mathbb{R}$ such that $f_{\alpha}\left(x_{\alpha}\right)-e_{\alpha} \geq \varepsilon>0$ and $\left|r-f_{\alpha}\left(x_{\alpha}\right)\right| \leq \varepsilon / 4$ for all $\alpha<\omega_{1}$. Hence, if $\alpha, \beta<\omega_{1}$ with $\alpha \neq \beta$, we have

$$
f_{\alpha}\left(x_{\alpha}\right) \geq r-\varepsilon / 4>r-3 \varepsilon / 4 \geq f_{\beta}\left(x_{\beta}\right)-\varepsilon \geq e_{\beta} \geq f_{\beta}\left(x_{\alpha}\right),
$$

which implies that $\left\{f_{\alpha}\right\}_{\alpha<\omega_{1}}$ is a $w^{*}-\omega_{1}$-polyhedron in $X^{*}$.

The converse implication is analogous.

In the following proposition we give the relation between $\omega_{1}$-polyhedrons and UBABS.

Proposition 2.2. For a Banach space $X$ the following are equivalent:

(1) $X$ has an $\omega_{1}$-polyhedron.

(2) $X$ has an UBABS of type $\eta$ for some $0 \leq \eta<1$.

(3) $X$ has an $U B A B S$.

Proof. $(1) \Rightarrow(2)$. If $w^{*}-\operatorname{Dens}\left(X^{*}\right) \geq \aleph_{0}$, then $X$ has an uncountable biorthogonal system and so $X$ has an UBABS of type 0 . Now assume that 
$w^{*}$-Dens $\left(X^{*}\right) \leq \aleph_{0}$. Let $\left\{x_{\alpha}\right\}_{1 \leq \alpha<\omega_{1}} \subset X$ be an $\omega_{1}$-polyhedron. Assume that $x_{1}=0$ and $\left\|x_{\alpha}\right\| \leq 1$. For each $1 \leq \alpha<\omega_{1}$ consider $f_{\alpha} \in S\left(X^{*}\right)$ such that

$$
1 \geq f_{\alpha}\left(x_{\alpha}\right)>\sup \left\{f_{\alpha}\left(x_{i}\right): 1 \leq i<\omega_{1}, i \neq \alpha\right\}=: \varrho_{\alpha} .
$$

Observe that $\varrho_{\alpha} \geq 0$ if $\alpha \neq 1$. By passing to an uncountable subsequence, it can be assumed that there are real numbers $0<\varepsilon, r \leq 1$ such that $f_{\alpha}\left(x_{\alpha}\right)-\varrho_{\alpha} \geq \varepsilon$ and $\left|r-f_{\alpha}\left(x_{\alpha}\right)\right|<\varepsilon / 8$ for every $2 \leq \alpha<\omega_{1}$. Since $w^{*}$ Dens $\left(X^{*}\right) \leq \aleph_{0}$, by passing again to a subsequence, we assume that there exists $z \in X^{*}$ such that $z\left(x_{\alpha}\right)>0$ and $\left|z\left(x_{\beta}\right) / z\left(x_{\alpha}\right)-1\right|<\varepsilon / 8$ for every $2 \leq \alpha, \beta<\omega_{1}$. Then, if $g_{\alpha}=f_{\alpha}+z / z\left(x_{\alpha}\right), 2 \leq \alpha<\omega_{1}$, we have

$$
\begin{aligned}
g_{\alpha}\left(x_{\alpha}\right) & =f_{\alpha}\left(x_{\alpha}\right)+1 \geq r-\varepsilon / 8+1>r-6 \varepsilon / 8+1 \geq f_{\alpha}\left(x_{\alpha}\right)-7 \varepsilon / 8+1 \\
& \geq \sup \left\{g_{\alpha}\left(x_{\beta}\right): 2 \leq \beta<\omega_{1}, \beta \neq \alpha\right\} \\
& \geq \inf \left\{g_{\alpha}\left(x_{\beta}\right): 2 \leq \beta<\omega_{1}, \beta \neq \alpha\right\} \geq-\varepsilon / 8 .
\end{aligned}
$$

Define $h_{\alpha}=g_{\alpha} / g_{\alpha}\left(x_{\alpha}\right)$. Then, for $2 \leq \alpha, \beta<\omega_{1}, \alpha \neq \beta$, we have $h_{\alpha}\left(x_{\alpha}\right)=1$ and

$$
-\frac{\varepsilon / 8}{r-\varepsilon / 8+1} \leq-\frac{\varepsilon / 8}{g_{\alpha}\left(x_{\alpha}\right)} \leq h_{\alpha}\left(x_{\beta}\right)=\frac{g_{\alpha}\left(x_{\beta}\right)}{g_{\alpha}\left(x_{\alpha}\right)} \leq \frac{r+1-6 \varepsilon / 8}{r+1-\varepsilon / 8} .
$$

So, $\left\{\left(x_{\alpha}, h_{\alpha}\right): 2 \leq \alpha<\omega_{1}\right\} \subset X \times X^{*}$ is an UBABS of type $\eta$ such that

$$
0 \leq \eta=\max \left\{\frac{\varepsilon / 8}{r-\varepsilon / 8+1}, \frac{r+1-6 \varepsilon / 8}{r+1-\varepsilon / 8}\right\}<1 .
$$

$(2) \Rightarrow(3)$ is obvious and $(3) \Rightarrow(1)$ is clear, because if $\left\{\left(x_{\alpha}, f_{\alpha}\right)\right\}_{1 \leq \alpha<\omega_{1}} \subset$ $X \times X^{*}$ is an UBABS, then $\left\{x_{\alpha}\right\}_{\alpha<\omega_{1}}$ is an $\omega_{1}$-polyhedron.

Let us consider some results on representation of elements in polyhedrons, which we need later. If $\left\{x_{i}\right\}_{i \in I}$ is a $w^{*}-\tau$-polyhedron in a dual Banach space $X^{*}$ with $\tau=\operatorname{card}(I)$ and $K=\overline{\mathrm{co}}^{w^{*}}\left(\left\{x_{i}\right\}_{i \in I}\right)$, then the core of $K$ is the set

$$
K_{0}=\operatorname{core}(K)=\bigcap\left\{\overline{\mathrm{co}}^{w^{*}}\left(\left\{x_{i}\right\}_{i \in I \backslash A}\right): A \subset I, A \text { finite }\right\} .
$$

Define the function $\lambda: K \rightarrow[0,1]$ as follows: for $k \in K$,

$$
\lambda(k)=\sup \left\{\lambda \in[0,1]: \exists u \in K, \exists i \in I \text { such that } k=\lambda x_{i}+(1-\lambda) u\right\} .
$$

Let $H=\{x \in K: \lambda(x)=0\}$. Since for every finite subset $A \subset I$, each $x \in K$ has the expression $x=\sum_{i \in A} \lambda_{i} x_{i}+(1-\mu) u$ with $u \in \overline{\mathrm{co}}^{w^{*}}\left(\left\{x_{i}\right\}_{i \in I \backslash A}\right)$, $\lambda_{i} \in[0,1], i \in A, \mu=\sum_{i \in A} \lambda_{i} \leq 1$, it can be easily seen that $H \subset K_{0}$.

LEMMA 2.3. Let $\left\{x_{i}\right\}_{i \in I}$ be a $w^{*}-\tau$-polyhedron in the dual Banach space $X^{*}, \tau=\operatorname{card}(I), K=\overline{\mathrm{co}}^{w^{*}}\left(\left\{x_{i}\right\}_{i \in I}\right), K_{0}=\operatorname{core}(K)$ and $H=\{x \in K$ : $\lambda(x)=0\}$. If $x \in K$, then there exist a sequence $\left\{\mu_{n}\right\}_{n \geq 1}$ of positive numbers with $0 \leq \sum_{n \geq 1} \mu_{n}=\mu \leq 1$, a sequence $\left\{i_{n}\right\}_{n \geq 1} \subset I$ of indices (not necessarily distinct) and $u \in H$ such that $x=\sum_{n \geq 1} \mu_{n} x_{i_{n}}+(1-\mu) u$. 
Proof. Clearly the statement is true if $x \in H$. Assume that $x \notin H$, i.e., $\lambda(x)>0$. Choose $0<\frac{1}{2} \lambda(x) \leq \lambda_{1} \leq 1, i_{1} \in I$, and $u_{1} \in \overline{\mathrm{co}} w^{*}\left(\left\{x_{i}\right\}_{i \in I \backslash\left\{i_{1}\right\}}\right)$ such that $x=\lambda_{1} x_{i_{1}}+\left(1-\lambda_{1}\right) u_{1}$. If $u_{1} \in H$, we are done. Otherwise, $\lambda\left(u_{1}\right)>0$ and we choose $0<\frac{1}{2} \lambda\left(u_{1}\right) \leq \lambda_{2} \leq 1, i_{2} \in I$, and $u_{2} \in \overline{\mathrm{CO}} w^{*}\left(\left\{x_{i}\right\}_{i \in I \backslash\left\{i_{2}\right\}}\right)$ such that $u_{1}=\lambda_{2} x_{i_{2}}+\left(1-\lambda_{2}\right) u_{2}$. By reiteration, there are two possibilities:

(A) $u_{m} \in H$ for some $m \in \mathbb{N}$. Then we obtain the representation

$$
x=\sum_{k=1}^{m} \lambda_{k} P_{k-1} x_{i_{k}}+P_{m} u_{m}, \quad P_{n}=\prod_{k=1}^{n}\left(1-\lambda_{k}\right), \quad P_{0}=1 .
$$

(B) Always $u_{m} \notin H$. As $P_{m}$ decreases in (1), the $\operatorname{limit}_{m \geq 1} P_{m}=P \in$ $[0,1]$ exists. We have two cases:

- $P>0$. Observe that this happens iff $\sum_{k \geq 1} \lambda_{k}<\infty$. In consequence, the series $\sum_{k \geq 1} \lambda_{k} P_{k-1} x_{i_{k}}$ converges and $u_{m} \stackrel{\rightarrow}{\rightarrow} u \in K$ as $m \rightarrow \infty$. So $x=\sum_{k \geq 1} \lambda_{k} P_{k-1} x_{i_{k}}+P u$. We claim that $\lambda(u)=0$. Indeed, suppose that $\mu:=\lambda(u)>0$ and pick $q \in \mathbb{N}$ such that $P / P_{q}>1 / 2, \lambda_{q+1}<\mu / 8$. Then

$$
u_{q}=\frac{1}{P_{q}}\left(\sum_{j \geq 1} \lambda_{q+j} P_{q+j-1} x_{q+j}+P u\right),
$$

which implies that $\lambda\left(u_{q}\right) \geq\left(P / P_{q}\right) \lambda(u)=\left(P / P_{q}\right) \mu>\mu / 2$. Since $0<$ $\frac{1}{2} \lambda\left(u_{q}\right) \leq \lambda_{q+1} \leq 1$, we obtain $\mu / 8>\lambda_{q+1} \geq \mu / 4$, a contradiction.

- $P=0$. In this case $P_{m} u_{m} \rightarrow 0$ as $m \rightarrow \infty$ and we obtain the representation $x=\sum_{k \geq 1} \lambda_{k} P_{k-1} x_{i_{k}}$ with $\sum_{k \geq 1} \lambda_{k} P_{k-1}=1$.

In order to connect the existence of an UBABS in a Banach space $X$ with the $w^{*}$-nonseparability of dual equivalent unit balls of $X^{*}$, we introduce the index $\sigma(X)$. If $K \subset X^{*}$ is a disc (i.e., a convex symmetric subset of $X^{*}$ ), define

$$
\sigma(K)=\max \left\{0 \leq t \leq 1: \exists A \subset K,|A| \leq \aleph_{0}, t K \subseteq \overline{\mathrm{co}}^{w^{*}}(A \cup(-A))\right\} .
$$

Observe that $0 \leq \sigma(K)<1$ iff $K$ is $w^{*}$-nonseparable and that there exists a countable subset $A \subset K$ such that $\sigma(K) \cdot K \subset \overline{\mathrm{co}}^{w^{*}}(A \cup(-A))$.

Lemma 2.4. Let $X$ be a Banach space, $K \subset X^{*}$ a $w^{*}$-nonseparable disc and $\sigma(K)<\varrho \leq 1$. Then there exists $\varepsilon=\varepsilon(\varrho)>0$ (depending on $\varrho$ ) such that for every countable subset $A \subset K$ there exists $k \in K$ satisfying $\operatorname{dist}\left(\varrho k, \overline{\mathrm{co}}^{w^{*}}(A \cup(-A))\right) \geq \varepsilon$.

Proof. In the contrary case, there exist a sequence of real numbers $\varepsilon_{n} \downarrow 0$ and a sequence of countable subsets $A_{n} \subset K, n \geq 1$, such that every $k \in K$ satisfies $\operatorname{dist}\left(\varrho k, \overline{\mathrm{co}} w^{*}\left(A_{n} \cup\left(-A_{n}\right)\right)\right)<\varepsilon_{n}$. So, if $A=\bigcup_{n \geq 1} A_{n}$ we have $\varrho K \subset \overline{\mathrm{co}} w^{*}(A \cup(-A))$, a contradiction.

Define the index $\sigma(X), X$ a Banach space, as follows:

$$
\sigma(X)=\inf \left\{\sigma(K): K \subset X^{*} \text { a dual equivalent ball of } X^{*}\right\} .
$$


It is clear that $\sigma(X)$ is invariant under isomorphisms.

Proposition 2.5. For a Banach space $X$ we have

$$
\sigma(X)=\inf \left\{\sigma(K): K \subset X^{*} \text { a } w^{*} \text {-compact disc }\right\} .
$$

Proof. Obviously $\sigma(X) \geq \inf \left\{\sigma(K): K \subset X^{*}\right.$ a $w^{*}$-compact disc $\}$. In order to prove the opposite inequality, it is enough to see that $\sigma(X) \leq \sigma(K)$ for any $w^{*}$-compact disc $K \subset X^{*}$. Assume that such a $K$ is $w^{*}$-nonseparable, pick $\sigma(K)<\varrho<1$ and let $\varepsilon=\varepsilon(\varrho)>0$ be given by Lemma 2.4. For $0<$ $\delta<\varepsilon$ such that $\varrho+\delta<1$ consider $H_{\delta}=K+\delta B\left(X^{*}\right)$, which is an equivalent dual ball of $X^{*}$. We claim that $\sigma\left(H_{\delta}\right) \leq \varrho+\delta$. Indeed, let $\varrho+\delta<t \leq 1$ and $A \subset H_{\delta}$ be a countable subset. Then $A \subset A_{1}+A_{2}$, where $A_{1} \subset K$ and $A_{2} \subset \delta B\left(X^{*}\right)$ are countable. Assume that $t H_{\delta} \subset \overline{\mathrm{co}} w^{*}(A \cup(-A))$. As $\overline{\mathrm{CO}}^{w^{*}}(A \cup(-A)) \subset \overline{\mathrm{co}} w^{*}\left(A_{1} \cup\left(-A_{1}\right)\right)+\delta B\left(X^{*}\right)$, we get

$$
t K \subset t H_{\delta} \subset \overline{\mathrm{co}}^{w^{*}}\left(A_{1} \cup\left(-A_{1}\right)\right)+\delta B\left(X^{*}\right),
$$

which implies that $\operatorname{dist}\left(t k, \overline{\mathrm{CO}}^{w^{*}}\left(A_{1} \cup\left(-A_{1}\right)\right)\right) \leq \delta$ for all $k \in K$. But by Lemma 2.4 there exists $k \in K$ such that $\operatorname{dist}\left(\varrho k, \overline{\mathrm{co}}^{w^{*}}\left(A_{1} \cup\left(-A_{1}\right)\right)\right) \geq \varepsilon$. Thus $\operatorname{dist}\left(t k, \overline{\mathrm{co}}^{w^{*}}\left(A_{1} \cup\left(-A_{1}\right)\right)\right)>\delta$, a contradiction. Therefore, we have $t H_{\delta} \nsubseteq \overline{\mathrm{co}} w^{*}(A \cup(-A))$ and $\sigma\left(H_{\delta}\right) \leq \varrho+\delta$ for $0<\delta<\varepsilon$. Hence, $\sigma(X) \leq \varrho$ for every $\sigma(K)<\varrho<1$, and we conclude that $\sigma(X) \leq \sigma(K)$.

Proposition 2.6. If $X$ is a Banach space then $\sigma(X) \leq \tau(X)$.

Proof. Assume that $\tau(X)<\eta<1$ and choose an UBABS $\left\{\left(x_{\alpha}, f_{\alpha}\right)\right\}_{\alpha<\omega_{1}}$ $\subset X \times X^{*}$ of type $\eta$ such that $\left\|f_{\alpha}\right\|=1$ and $\left\|x_{\alpha}\right\| \leq M$ for all $\alpha<\omega_{1}$, for some $0<M<\infty$. Clearly, $\left\{ \pm f_{\alpha}\right\}_{\alpha<\omega_{1}}$ is a $w^{*}-\omega_{1}$-polyhedron. Define $K=\overline{\mathrm{co}} w^{*}\left(\left\{ \pm f_{\alpha}\right\}_{\alpha<\omega_{1}}\right), K_{0}=\operatorname{core}(K)$ and $H=\{z \in K: \lambda(z)=0\}$. It is easy to see that $\left|z\left(x_{\alpha}\right)\right| \leq \eta$ for every $z \in K_{0}$ and $\alpha<\omega_{1}$. We claim that $\sigma(K) \leq \eta$. Indeed, let $A \subset K$ be countable. By Lemma 2.3 there exists $\gamma<\omega_{1}$ such that

$$
A \subset \overline{\mathrm{co}}\left(\left\{ \pm f_{\alpha}\right\}_{\alpha \leq \gamma} \cup H\right) \subset \overline{\mathrm{co}^{w}} w^{*}\left(\left\{ \pm f_{\alpha}\right\}_{\alpha \leq \gamma} \cup H\right) .
$$

Clearly, $\overline{\mathrm{co}} w^{*}(A \cup(-A)) \subset \overline{\mathrm{co}} w^{*}\left(\left\{ \pm f_{\alpha}\right\}_{\alpha \leq \gamma} \cup H\right)$ and for every $\gamma<\varrho<\omega_{1}$ and every $z \in \overline{\mathrm{CO}} w^{*}\left(\left\{ \pm f_{\alpha}\right\}_{\alpha \leq \gamma} \cup H\right)$ we have $\left|z\left(x_{\varrho}\right)\right| \leq \eta$.

Hence, for every $\gamma<\varrho<\omega_{1}$ and $\eta<t \leq 1$ we have $t f_{\varrho} \notin \overline{\mathrm{co}^{w^{*}}}(A \cup(-A))$. So $\sigma(K) \leq \eta$ and we conclude that $\sigma(X) \leq \tau(X)$.

Now we prove for a Banach space $X$ that $\sigma(X)=1$ iff $\tau(X)=1$.

Proposition 2.7. A Banach space $X$ has an UBABS of type $\eta$ for some $\eta \in[0,1)$ iff $X^{*}$ has a $w^{*}$-nonseparable equivalent dual unit ball. So, $\sigma(X)=1$ iff $\tau(X)=1$.

Proof. Firstly, if $X$ has an UBABS of type $\eta$ for some $\eta \in[0,1)$ (i.e., $\tau(X)<1$ ), then by Proposition 2.6 we have $\sigma(X)<1$ (i.e., $X^{*}$ has a $w^{*}$-nonseparable equivalent dual unit ball). 
Assume now that $X$ is a Banach space with $\sigma(X)<1$ equipped with an equivalent norm such that $\sigma\left(B\left(X^{*}\right)\right)<1$. Fix $\varrho>0$ with $\sigma\left(B\left(X^{*}\right)\right)<\varrho<1$. If $A \subset S(X)$ and $\varepsilon \geq 0$ we put

$$
(A, \varepsilon)^{\perp}=\left\{z \in X^{*}:|z(x)| \leq \varepsilon, \forall x \in A\right\}, \quad S\left((A, \varepsilon)^{\perp}\right)=S\left(X^{*}\right) \cap(A, \varepsilon)^{\perp} .
$$

Clearly $\varepsilon B\left(X^{*}\right)+A^{\perp} \subset(A, \varepsilon)^{\perp}$.

Claim 0. If $A \subset S(X)$ and $A^{\perp} \neq\{0\}$, then $\varepsilon S\left(X^{*}\right) \subset \operatorname{co}\left(S\left((A, \varepsilon)^{\perp}\right)\right)$ for $0 \leq \varepsilon<1$.

Indeed, let $u \in \varepsilon S\left(X^{*}\right)$ and $v \in A^{\perp} \backslash\{0\}$. We can find $\lambda, \mu>0$ such that $u+\lambda v, u-\mu v \in S\left(X^{*}\right)$. Thus, $u+\lambda v, u-\mu v \in S\left((A, \varepsilon)^{\perp}\right)$. Let $t \in(0,1)$ be such that $t \lambda+(1-t)(-\mu)=0$. Then $u=t(u+\lambda v)+(1-t)(u-\mu v) \in$ $\operatorname{co}\left(S\left((A, \varepsilon)^{\perp}\right)\right)$.

Claim 1. For any countable subsets $A \subset S(X)$ and $F \subset S\left(X^{*}\right)$ there exists $f \in S\left((A, \sqrt{\varrho})^{\perp}\right)$ such that $\sqrt{\varrho} f \notin \overline{\mathrm{co}^{w^{*}}}(F \cup(-F))$.

The opposite means that $\sqrt{\varrho} S\left((A, \sqrt{\varrho})^{\perp}\right) \subset \overline{\mathrm{co}}^{w^{*}}(F \cup(-F))$. By Claim 0 we have $\sqrt{\varrho} S\left(X^{*}\right) \subset \operatorname{co}\left(S\left((A, \sqrt{\varrho})^{\perp}\right)\right)$. So

$$
\varrho B\left(X^{*}\right) \subset \overline{\mathrm{co}}^{w^{*}}\left(\varrho S\left(X^{*}\right)\right) \subset \overline{\mathrm{co}}^{w^{*}}\left(\sqrt{\varrho} S\left((A, \sqrt{\varrho})^{\perp}\right)\right) \subset \overline{\mathrm{co}}^{w^{*}}(F \cup(-F)),
$$

a contradiction because $\sigma\left(B\left(X^{*}\right)\right)<\varrho$. So, Claim 1 holds.

Claim 2. There exist $0 \leq \delta<\varepsilon \leq 1-\sqrt{\varrho}$ such that for any countable subsets $A \subset S(X)$ and $F \subset S\left(X^{*}\right)$ there exist $f_{0} \in S\left((A, \sqrt{\varrho})^{\perp}\right)$ and $x_{0} \in$ $S(X)$ such that $f_{0}\left(x_{0}\right) \geq 1-\delta$ and $f\left(x_{0}\right) \leq 1-\varepsilon$ for all $f \in F$.

Define $\mathcal{R}=\left\{r=\left(r_{1}, r_{2}\right) \in \mathbb{Q} \times \mathbb{Q}: 0<r_{1}<r_{2} \leq 1-\sqrt{\varrho}\right\}$. As $\mathcal{R}$ is countable, we can put $\mathcal{R}=\left\{r_{n}\right\}_{n \geq 1}$. If Claim 2 is false, for every pair $r_{n}=\left(r_{n 1}, r_{n 2}\right) \in \mathcal{R}$ we can choose countable subsets $A_{n} \subset S(X), F_{n} \subset$ $S\left(X^{*}\right), n \geq 1$, such that for every $g \in S\left(\left(A_{n}, \sqrt{\varrho}\right)^{\perp}\right)$ and every $x \in S(X)$, either $g(x)<1-r_{n 1}$ or there exists $f \in F_{n}$ with $f(x)>1-r_{n 2}$. Let $A=\bigcup_{n \geq 1} A_{n}, F=\bigcup_{n \geq 1} F_{n}$. By Claim 1 there exists $f_{0} \in S\left((A, \sqrt{\varrho})^{\perp}\right)$ such that $\sqrt{\varrho} f_{0} \notin \overline{\mathrm{Co}^{2}} w^{*}(F \cup(-F))$. By the Hahn-Banach Theorem there exists $y \in S(X)$ such that

$$
\sqrt{\varrho} f_{0}(y)>\sup \{|f(y)|: f \in F\}=: \gamma_{0} \geq 0 .
$$

Choose a sequence $\left\{z_{n}\right\}_{n \geq 1} \subset S(X)$ such that

$$
\lim _{n \rightarrow \infty} f_{0}\left(z_{n}\right)=\left\|f_{0}\right\|=1, \quad 1-f_{0}\left(z_{n}\right)<\frac{1}{n}\left(f_{0}(y)-\gamma_{0}\right), \quad n \geq 1 .
$$

Then

$$
f_{0}\left(\frac{z_{n}+\frac{1}{n} y}{\left\|z_{n}+\frac{1}{n} y\right\|}\right)=1-\delta_{n}
$$


with

$$
0 \leq \delta_{n}=\frac{\left\|z_{n}+\frac{1}{n} y\right\|-f_{0}\left(z_{n}\right)-\frac{1}{n} f_{0}(y)}{\left\|z_{n}+\frac{1}{n} y\right\|} \leq \frac{1-f_{0}\left(z_{n}\right)+\frac{1}{n}\left(1-f_{0}(y)\right)}{\left\|z_{n}+\frac{1}{n} y\right\|} .
$$

Hence, $\lim _{n \rightarrow \infty} \delta_{n}=0$. On the other hand, for every $f \in F$,

$$
f\left(\frac{z_{n}+\frac{1}{n} y}{\left\|z_{n}+\frac{1}{n} y\right\|}\right) \leq \frac{1+\frac{1}{n} \gamma_{0}}{\left\|z_{n}+\frac{1}{n} y\right\|}=1-\varepsilon_{n},
$$

where

$$
\varepsilon_{n}=\frac{\left\|z_{n}+\frac{1}{n} y\right\|-1-\frac{1}{n} \gamma_{0}}{\left\|z_{n}+\frac{1}{n} y\right\|} \leq \frac{1+\frac{1}{n}-1-\frac{1}{n} \gamma_{0}}{\left\|z_{n}+\frac{1}{n} y\right\|}=\frac{\frac{1}{n}\left(1-\gamma_{0}\right)}{\left\|z_{n}+\frac{1}{n} y\right\|}
$$

and

$$
\varepsilon_{n}>\frac{\left\|z_{n}+\frac{1}{n} y\right\|-f_{0}\left(z_{n}\right)-\frac{1}{n} f_{0}(y)}{\left\|z_{n}+\frac{1}{n} y\right\|}=\delta_{n} \geq 0
$$

by (2). Pick any $n \in \mathbb{N}$ such that $\frac{1}{n}\left(1-\gamma_{0}\right) /\left\|z_{n}+\frac{1}{n} y\right\| \leq 1-\sqrt{\varrho}$. Then $0 \leq \delta_{n}<\varepsilon_{n} \leq 1-\sqrt{\varrho}$ and there is some $m \in \mathbb{N}$ such that $\delta_{n} \leq r_{m 1}<$ $r_{m 2} \leq \varepsilon_{n}$. Let $x_{0}=\left(z_{n}+\frac{1}{n} y\right) /\left\|z_{n}+\frac{1}{n} y\right\| \in S(X)$ and observe that $f_{0} \in$ $S\left(\left(A_{m}, \sqrt{\varrho}\right)^{\perp}\right), f_{0}\left(x_{0}\right) \geq 1-\delta_{n}$ and $f\left(x_{0}\right) \leq 1-\varepsilon_{n}$ for all $f \in F$. Then $f_{0} \in S\left(\left(A_{m}, \sqrt{\varrho}\right)^{\perp}\right), f_{0}\left(x_{0}\right) \geq 1-r_{m 1}$ and $f\left(x_{0}\right) \leq 1-\varepsilon_{n} \leq 1-r_{m 2}$ for all $f \in F_{m}$, a contradiction. So, Claim 2 holds.

Let $0 \leq \delta<\varepsilon \leq 1-\sqrt{\varrho}$ be from Claim 2. We will construct a transfinite sequence $\left\{\left(x_{\alpha}, f_{\alpha}\right)\right\}_{\alpha<\omega_{1}} \subset S(X) \times S\left(X^{*}\right)$ so that for every $\alpha<\omega_{1}$,

$$
\begin{aligned}
& f_{\alpha}\left(x_{\alpha}\right) \geq 1-\delta, \\
& f_{\alpha}\left(x_{\beta}\right) \leq 1-\varepsilon \quad \text { if } \alpha \neq \beta .
\end{aligned}
$$

In the first step, we take $x_{1} \in S(X)$ and $f_{1} \in S\left(X^{*}\right)$ such that $f_{1}\left(x_{1}\right)=1$. Let $1<\alpha_{0}<\omega_{1}$ and suppose we have constructed a family $\left\{\left(x_{\alpha}, f_{\alpha}\right)\right.$ : $\left.\alpha<\alpha_{0}\right\}$ satisfying (3) and (4). Apply Claim 2, with $F=\left\{f_{\alpha}: \alpha<\alpha_{0}\right\}$ and $A=\left\{x_{\alpha}: \alpha<\alpha_{0}\right\}$. Denote the resulting elements $x_{0}$ and $f_{0}$ by $x_{\alpha_{0}}$ and $f_{\alpha_{0}}$. The inequality (3) for $\alpha=\alpha_{0}$ is satisfied by construction. The inequality (4) for $\alpha=\alpha_{0}$ and $\beta<\alpha_{0}$ holds because $f_{0} \in S\left((A, \sqrt{\varrho})^{\perp}\right)$ and $\varepsilon \leq 1-\sqrt{\varrho}$. For $\beta=\alpha_{0}$ and $\alpha<\alpha_{0}$, it follows because $\sup \left\{f\left(x_{0}\right): f \in F\right\} \leq 1-\varepsilon$. Now the set $\left\{\left(\bar{x}_{\alpha}, \bar{f}_{\alpha}\right)\right\}_{\alpha<\omega_{1}}$, where $\bar{x}_{\alpha}=x_{\alpha}, \bar{f}_{\alpha}=f_{\alpha} / f_{\alpha}\left(x_{\alpha}\right), 1 \leq \alpha<\omega_{1}$, is an uncountable bounded (by $(1-\delta)^{-1}$ ) almost biorthogonal system.

Proposition 2.8. Let $X$ be a Banach space such that $\sigma(X)<1 / 3$. Then $\tau(X) \leq 2 \sigma(X) /(1-\sigma(X))$. So, for every Banach space $X$ :

(1) $\sigma(X)=0$ iff $\tau(X)=0$.

(2) $\sigma(X)=0$ whenever $X$ has an uncountable biorthogonal system.

Proof. (A) Let $\|\cdot\|$ be an equivalent norm on $X$ such that the corresponding dual unit ball $B\left(X^{*}\right)$ satisfies $\sigma\left(B\left(X^{*}\right)\right)<1 / 3$. It is enough to 
prove that for every $\sigma\left(B\left(X^{*}\right)\right)<a<1 / 3$ there exists in $X$ an UBABS of type $\eta \leq 2 a /(1-a)$. So, fix such an $a$. By induction we choose a family $\left\{\left(x_{\alpha}, f_{\alpha}\right)\right\}_{\alpha<\omega_{1}} \subset S(X) \times S\left(X^{*}\right)$ such that

$$
f_{\alpha}\left(x_{\alpha}\right)>\frac{1-a}{2}, \quad\left|f_{\alpha}\left(x_{\beta}\right)\right|<a \quad \text { if } \alpha \neq \beta \text {. }
$$

Pick $\left(x_{1}, f_{1}\right) \in S(X) \times S\left(X^{*}\right)$ satisfying $f_{1}\left(x_{1}\right)=1$. Let $\alpha<\omega_{1}$ and assume that we have chosen $\left\{\left(x_{\beta}, f_{\beta}\right)\right\}_{\beta<\alpha} \subset S(X) \times S\left(X^{*}\right)$ satisfying (5). Set

$$
A_{\alpha}=\overline{\left[\left\{x_{\beta}: \beta<\alpha\right\}\right]}, \quad F_{\alpha}=\overline{\mathrm{co}} w^{*}\left(\left\{ \pm f_{\beta}: \beta<\alpha\right\} \cup G_{0}\right),
$$

where $G_{0} \subset B\left(X^{*}\right)$ is a countable symmetric subset 1-norming on $A_{\alpha}$. By $\left[15\right.$, Lemma 4.3] there exists $x_{\alpha} \in S(X)$ such that $\sup \left\{\left|f\left(x_{\alpha}\right)\right|: f \in F_{\alpha}\right\}<a$. We claim that $\operatorname{dist}\left(x_{\alpha}, A_{\alpha}\right)>(1-a) / 2$. Indeed, pick $z \in A_{\alpha}$ and observe that if $\|z\|<(1+a) / 2$, then clearly $\left\|z-x_{\alpha}\right\|>(1-a) / 2$, and if $\|z\| \geq$ $(1+a) / 2$, then

$$
\begin{aligned}
\left\|z-x_{\alpha}\right\| & \geq \sup \left\{f\left(z-x_{\alpha}\right): f \in F_{\alpha}\right\} \\
& \geq\|z\|-\sup \left\{f\left(x_{\alpha}\right): f \in F_{\alpha}\right\}>\frac{1+a}{2}-a=\frac{1-a}{2} .
\end{aligned}
$$

This means that if $Q: X \rightarrow X / A_{\alpha}$ is the canonical quotient mapping, then $\left\|Q\left(x_{\alpha}\right)\right\|>(1-a) / 2$. So, as $\left(X / A_{\alpha}\right)^{*}=A_{\alpha}^{\perp}$ there exists $f_{\alpha} \in S\left(X^{*}\right) \cap A_{\alpha}^{\perp}$ such that $f_{\alpha}\left(x_{\alpha}\right)>(1-a) / 2$. Thus we have chosen the pair $\left(x_{\alpha}, f_{\alpha}\right)$, and this completes the induction.

Now put $\widetilde{f}_{\alpha}=f_{\alpha} / f_{\alpha}\left(x_{\alpha}\right)$, consider the family $\mathfrak{F}=\left\{\left(x_{\alpha}, \widetilde{f}_{\alpha}\right)\right\}_{\alpha<\omega_{1}}$ and observe that:

(a) $\mathfrak{F}$ is bounded because $\left\|x_{\alpha}\right\|=1$ and

$$
\left\|\tilde{f}_{\alpha}\right\|=\frac{\left\|f_{\alpha}\right\|}{\left|f_{\alpha}\left(x_{\alpha}\right)\right|}<\frac{1}{(1-a) / 2}=\frac{2}{1-a}<\frac{2}{1-1 / 3}=3 .
$$

(b) $\tilde{f}_{\alpha}\left(x_{\alpha}\right)=1$ and

$$
\left|\tilde{f}_{\alpha}\left(x_{\beta}\right)\right|=\frac{\left|f_{\alpha}\left(x_{\beta}\right)\right|}{f_{\alpha}\left(x_{\alpha}\right)}<\frac{a}{(1-a) / 2}=\frac{2 a}{1-a}<1 \quad \text { if } \alpha \neq \beta .
$$

So, $\mathfrak{F}$ is an UBABS of type $\eta \leq 2 a /(1-a)$.

(B) (1) follows from (A) and Proposition 2.6; (2) follows from the definition of $\tau(X)$ and (1).

3. On $\omega$-independence. The Kunen-Shelah property $\mathrm{KS}_{3}$. A family $\left\{x_{i}\right\}_{i \in I}$ in a Banach space $X$ is said to be $\omega$-independent if for every sequence $\left(i_{n}\right)_{n \geq 1} \subset I$ of distinct indices, and every sequence $\left(\lambda_{n}\right)_{n \geq 1} \subset \mathbb{R}$, the series $\sum_{n=1}^{\infty} \bar{\lambda}_{n} x_{i_{n}}$ converges (in norm) to 0 iff $\lambda_{n}=0$ for every $n \geq 1$ (see $[6],[12])$. A Banach space $X$ is said to have the Kunen-Shelah property $\mathrm{KS}_{3}$ 
if $X$ has no uncountable $\omega$-independent family. Of course, every biorthogonal family is $\omega$-independent (i.e., $\mathrm{KS}_{3} \Rightarrow \mathrm{KS}_{2}$ ), but there are $\omega$-independent families which are not merely biorthogonal systems. Here is an example: $X=C\left([0,1]^{\omega_{1}}\right)$ and $\left\{f_{\alpha}^{n}\right\}_{\alpha<\omega_{1}, n \geq 1}$ defined as

$$
f_{\alpha}^{n}\left(\left(t_{\gamma}\right)_{\gamma<\omega_{1}}\right)=t_{\alpha}^{n}
$$

for every $x=\left(t_{\gamma}\right)_{\gamma<\omega_{1}} \in[0,1]^{\omega_{1}}$. This family is $\omega$-independent but not a biorthogonal system by the Theorem of Müntz-Szasz (see [11, Th. 15.26]).

QUESTION 2. Does a Banach space have an uncountable biorthogonal system whenever it has an uncountable $\omega$-independent family?

Unfortunately, the indices $\sigma(X), \tau(X)$ do not separate the properties $\mathrm{KS}_{2}$ and $\mathrm{KS}_{3}$, because as we prove in the following, if $X \in \mathrm{KS}_{3}$, then $\sigma(X)=0$.

Lemma 3.1. Let $X$ be a Banach space, $\left\{x_{i}\right\}_{1 \leq i<\omega_{1}} \subset X$ an uncountable bounded $\omega$-independent family, $H \subset X$ a closed separable subspace and $N \in \mathbb{N}$. Then there exist ordinal numbers $\varrho<\gamma<\omega_{1}$ such that $x_{\varrho} \notin$ $\overline{\mathrm{co}}\left(H \cup\left\{ \pm N x_{i}\right\}_{\gamma \leq i<\omega_{1}}\right)$.

Proof. Without loss of generality suppose that $\left\|x_{i}\right\| \leq 1$ for all $i<\omega_{1}$. Assume that for every pair of ordinal numbers $\varrho, \gamma$ such that $\varrho<\gamma<\omega_{1}$ we have $x_{\varrho} \in \overline{\mathrm{co}}\left(H \cup\left\{ \pm N x_{i}\right\}_{\gamma \leq i<\omega_{1}}\right)$. For $n \in \mathbb{N}$ and $\varrho<\gamma<\omega_{1}$, define $D_{\gamma}=\operatorname{co}\left(\left\{ \pm N x_{i}\right\}_{\gamma \leq i<\omega_{1}}\right)$ and $H(\varrho, \gamma, n)$

$$
=\left\{(u, \lambda) \in H \times(0,1]: \exists v \in D_{\gamma} \text { with }\left\|\lambda u+(1-\lambda) v-x_{\varrho}\right\|<\frac{1}{2 n}\right\} .
$$

If $\varrho<\gamma<\gamma^{\prime}<\omega_{1}$ and $n \geq 1$, then by hypothesis and definition, we have $H(\varrho, \gamma, n) \neq \emptyset$ and $H(\varrho, \gamma, n+1) \subset H(\varrho, \gamma, n) \supset H\left(\varrho, \gamma^{\prime}, n\right)$.

For $\beta<\omega_{1}$ and $n \geq 1$ define

$$
H(\beta, n)=\operatorname{cl}\left(\bigcup\left\{H(\varrho, \gamma, n): \beta \leq \varrho<\gamma<\omega_{1}\right\}\right)
$$

where "cl" means closure in $H \times(0,1]$. Clearly, for $\beta<\beta^{\prime}$ and $n \geq 1$ we have

$$
\emptyset \neq H\left(\beta^{\prime}, n\right) \subset H(\beta, n) \supset H(\beta, n+1) .
$$

Since $H \times(0,1]$ is hereditarily Lindelöf, for each $n \geq 1$ there exists $\beta_{n}<\omega_{1}$ such that for every $\beta_{n} \leq \beta<\omega_{1}$ we have $H(\beta, n)=H\left(\beta_{n}, n\right)$. So, for every $(u, \lambda) \in H\left(\beta_{n}, n\right)$ and every $\beta_{n} \leq \beta<\omega_{1}$ we have $(u, \lambda) \in H(\beta, n)$, which implies that there exist $\beta \leq \varrho<\gamma<\omega_{1}$ and $v \in D_{\gamma}$ such that

$$
\left\|x_{\varrho}-(\lambda u+(1-\lambda) v)\right\|<1 / n \text {. }
$$

Let $\beta_{0}=\sup _{n \geq 1} \beta_{n}$ and fix $\beta_{0} \leq \varrho<\gamma<\omega_{1}$ and $n \geq 1$. Pick $(u, \mu) \in$ $H(\varrho, \gamma, n)$ and $w \in D_{\gamma}$ such that $\left\|x_{\varrho}-(\mu u+(1-\mu) w)\right\|<1 /(2 n)$. Since 
$(u, \mu) \in H\left(\beta_{0}, n\right)=H(\gamma, n)$, there exist $\gamma \leq \sigma<\theta<\omega_{1}$ and $v \in D_{\theta}$ such that $\left\|x_{\sigma}-(\mu u+(1-\mu) v)\right\|<1 / n$.

Set $T=x_{\sigma}-(\mu u+(1-\mu) v)$. Then $\mu u=x_{\sigma}-T-(1-\mu) v$ and

$$
\left\|x_{\varrho}-\left(x_{\sigma}-T-(1-\mu) v+(1-\mu) w\right)\right\|<\frac{1}{2 n} .
$$

Since $\|T\|<1 / n$, we obtain

$$
\begin{aligned}
\left\|x_{\varrho}-\left(x_{\sigma}-(1-\mu) v+(1-\mu) w\right)\right\| & \\
& =\left\|x_{\varrho}-\left(x_{\sigma}-T-(1-\mu) v+(1-\mu) w\right)-T\right\| \\
& \leq\left\|x_{\varrho}-\left(x_{\sigma}-T-(1-\mu) v+(1-\mu) w\right)\right\|+\|T\|<\frac{1}{2 n}+\frac{1}{n}=\frac{3}{2 n} .
\end{aligned}
$$

Since $x_{\sigma}, v, w \in E_{\gamma}:=\overline{\left[\left\{x_{i}\right\}_{\gamma \leq i<\omega_{1}}\right]}$, letting $n \rightarrow \infty$ (with $\varrho, \gamma$ fixed) we deduce that $x_{\varrho} \in E_{\gamma}$ (in particular, this implies that $E_{\beta_{0}}=E_{\beta}$ for all $\left.\beta_{0} \leq \beta<\omega_{1}\right)$. Set $S=x_{\varrho}-\left(x_{\sigma}-(1-\mu) v+(1-\mu) w\right)$. Then

$$
x_{\varrho}=S+\mu v+(1-\mu) w+x_{\sigma}-v .
$$

Taking into account that $\mu v+(1-\mu) w,-v \in D_{\gamma}, x_{\sigma} \in(1 / N) D_{\gamma}$ and that $\|S\|<3 /(2 n)$, we finally get $x_{\varrho} \in \operatorname{cl}\left((1+1 / N) D_{\gamma}+D_{\gamma}\right)=\operatorname{cl}\left((2+1 / N) D_{\gamma}\right)$. So, $x_{\varrho}$ is an accumulation point of $F_{\gamma}:=(2+1 / N) D_{\gamma}$ (because $\left.x_{\varrho} \in \bar{F}_{\gamma} \backslash F_{\gamma}\right)$.

In consequence, we can conclude that every $x_{i}, \beta_{0} \leq i<\omega_{1}$, is an accumulation point of every $F_{\gamma}$ for $\gamma<\omega_{1}$.

Let $\left(a_{n}\right)_{n \geq 1}$ be a sequence of positive numbers such that $\lim _{n \rightarrow \infty} a_{n}=0$, $\sum_{n \geq 1} a_{n}=\infty$, and let $b_{n}=\sup _{m>n} a_{m}$. Fix $\beta_{0}<\tau<\omega_{1}$. Using the proof of [6, Th. 3], as in [12], we can construct inductively a sequence $\left\{\varepsilon_{n}\right\}_{n \geq 1}$ of signs, a sequence $\left\{\lambda_{r}^{n}\right\}_{n \geq 1,1 \leq r \leq k(n)}$ of real numbers and a sequence $\left\{\gamma_{r}^{n}\right\}_{n \geq 1,1 \leq r \leq k(n)}$ of ordinals such that:

(1) $\sum_{r=1}^{k(n)}\left|\lambda_{r}^{n}\right| \leq 2 N+1$ for every $n \geq 1$.

(2) $\tau<\gamma_{1}^{n}<\ldots<\gamma_{k(n)}^{n}<\gamma_{1}^{n+1}<\ldots<\omega_{1}$ for every $n \geq 1$.

(3) $x_{\tau}+\sum_{n \geq 1} a_{n} \varepsilon_{n} y_{n}=0$, where $y_{n}=\sum_{r=1}^{k(n)} \lambda_{r}^{n} x_{\gamma_{r}^{n}}$.

Let us see the first two steps of this argument. Set $K=\left\{x_{i}\right\}_{\tau<i<\omega_{1}}$.

STEP 1. By the proof of $\left[6\right.$, Th. 3] we can find $p_{1} \in \mathbb{N}$, a finite sequence $\left\{h_{n}\right\}_{1 \leq n \leq p_{1}}$ of (not necessarily distinct) elements of $K$ and a finite sequence $\left\{\varepsilon_{n}\right\}_{1 \leq n \leq p_{1}}$ of signs such that

$$
\begin{gathered}
\left\|x_{\tau}+\sum_{n=1}^{p_{1}} a_{n} \varepsilon_{n} h_{n}\right\|<2^{-1}, \\
\left\|x_{\tau}+\sum_{n=1}^{j} a_{n} \varepsilon_{n} h_{n}\right\|<b_{1}+1+2^{-1} \quad \text { for } 1 \leq j \leq p_{1} .
\end{gathered}
$$


Since $h_{n} \in \operatorname{cl}\left(F_{\beta}\right)$ for $\beta_{0} \leq \beta<\omega_{1}$, we can find, for $1 \leq n \leq p_{1}$, real numbers $\left\{\lambda_{r}^{n}\right\}_{1 \leq r \leq k(n)}$ with $\sum_{r=1}^{k(n)}\left|\lambda_{r}^{n}\right| \leq 2 N+1$, and ordinals $\left\{\gamma_{r}^{n}\right\}_{r=1}^{k(n)}$ such that:

(a) $\tau<\gamma_{1}^{n}<\ldots<\gamma_{k(n)}^{n}<\gamma_{1}^{n+1}<\ldots<\omega_{1}$.

(b) $\left\|x_{\tau}+\sum_{n=1}^{p_{1}} a_{n} \varepsilon_{n}\left(\sum_{r=1}^{k(n)} \lambda_{r}^{n} x_{\gamma_{r}^{n}}\right)\right\|<2^{-1}$.

(c) $\left\|x_{\tau}+\sum_{n=1}^{j} a_{n} \varepsilon_{n}\left(\sum_{r=1}^{k(n)} \lambda_{r}^{n} x_{\gamma_{r}^{n}}\right)\right\|<b_{1}+1+2^{-1}$ for $1 \leq j \leq p_{1}$.

STEP 2. Let $u_{1}=x_{\tau}+\sum_{n=1}^{p_{1}} a_{n} \varepsilon_{n}\left(\sum_{r=1}^{k(n)} \lambda_{r}^{n} x_{\gamma_{r}^{n}}\right)$. By the proof of $[6$, Th. 3] we can find $p_{1}<p_{2} \in \mathbb{N}$, a finite sequence $\left\{h_{n}\right\}_{p_{1}+1 \leq n \leq p_{2}}$ of (not necessarily distinct) elements of $K$ and a finite sequence $\left\{\varepsilon_{n}\right\}_{p_{1}+1 \leq n \leq p_{2}}$ of signs such that

$$
\begin{gathered}
\left\|u_{1}+\sum_{n=p_{1}+1}^{p_{2}} a_{n} \varepsilon_{n} h_{n}\right\|<2^{-2}, \\
\left\|u_{1}+\sum_{n=p_{1}+1}^{j} a_{n} \varepsilon_{n} h_{n}\right\|<b_{p_{1}}+2^{-1}+2^{-2} \quad \text { for } p_{1}+1 \leq j \leq p_{2} .
\end{gathered}
$$

Since $h_{n} \in \operatorname{cl}\left(F_{\beta}\right)$ for $\beta_{0} \leq \beta<\omega_{1}$, we can find, for $p_{1}<n \leq p_{2}$, real numbers $\left\{\lambda_{r}^{n}\right\}_{1 \leq r \leq k(n)}$ with $\sum_{r=1}^{k(n)}\left|\lambda_{r}^{n}\right| \leq 2 N+1$, and ordinals $\left\{\gamma_{r}^{n}\right\}_{r=1}^{k(n)}$ such that:

(a) $\gamma_{k\left(p_{1}\right)}^{p_{1}}<\gamma_{1}^{n}<\ldots<\gamma_{k(n)}^{n}<\gamma_{1}^{n+1}<\ldots<\omega_{1}$.

(b) $\left\|u_{1}+\sum_{n=p_{1}+1}^{p_{2}} a_{n} \varepsilon_{n}\left(\sum_{r=1}^{k(n)} \lambda_{r}^{n} x_{\gamma_{r}^{n}}\right)\right\|<2^{-2}$.

(c) $\left\|u_{1}+\sum_{n=p_{1}+1}^{j} a_{n} \varepsilon_{n}\left(\sum_{r=1}^{k(n)} \lambda_{r}^{n} x_{\gamma_{r}^{n}}\right)\right\|<b_{p_{1}}+2^{-1}+2^{-2}$ for $p_{1}<j \leq p_{2}$.

Now by reiteration we obtain the complete construction. It is easy to see that the series $x_{\tau}+\sum_{n \geq 1} a_{n} \varepsilon_{n}\left(\sum_{r=1}^{k(n)} \lambda_{r}^{n} x_{\gamma_{r}^{n}}\right)$ converges to zero. This proves that $\left\{x_{i}\right\}_{i<\omega_{1}}$ is not $\omega$-independent, a contradiction. So, we can choose $\varrho<\gamma<\omega_{1}$ such that $x_{\varrho} \notin \overline{\mathrm{co}}\left(H \cup\left\{ \pm N x_{i}\right\}_{\gamma \leq i<\omega_{1}}\right)$.

Proposition 3.2. Let a Banach space $X$ have an uncountable $\omega$-independent family $\left\{x_{\alpha}\right\}_{1 \leq \alpha<\omega_{1}}$. Then for every $0<\eta<1$, there exist an uncountable subsequence $\left\{\alpha_{i}\right\}_{i<\omega_{1}} \subset \omega_{1}$ and an $\operatorname{UBABS}\left\{\left(z_{i}, f_{i}\right)\right\}_{i<\omega_{1}} \subset$ $X \times X^{*}$ of type $\eta$ such that $z_{i}=x_{\alpha_{i}}$ and $f_{i}\left(z_{j}\right)=0$ for $j<i<\omega_{1}$. So, $\tau(X)=0$ and $X$ has an $\omega_{1}$-polyhedron.

Proof. Let $\left\{x_{i}\right\}_{1 \leq i<\omega_{1}} \subset X$ be an uncountable $\omega$-independent family and suppose, without loss of generality, that $\left\|x_{i}\right\| \leq 1$ for every $i<\omega_{1}$. Let $N \in \mathbb{N}$ be such that $1 / N \leq \eta$. In the following we choose by induction two subsequences $\left\{i_{\alpha}, j_{\alpha}\right\}_{\alpha<\omega_{1}}$ of ordinal numbers, with $i_{\alpha}<j_{\alpha} \leq i_{\beta}<j_{\beta}<\omega_{1}$ for $\alpha<\beta<\omega_{1}$, such that

$$
x_{i_{\alpha}} \notin \overline{\mathrm{co}}\left(\overline{\left[\left\{x_{i_{\beta}}: \beta<\alpha\right\}\right]} \cup\left\{ \pm N x_{j}\right\}_{j_{\alpha} \leq j<\omega_{1}}\right) .
$$


Indeed, let $\alpha<\omega_{1}$ and assume that we have chosen $\left\{i_{\beta}, j_{\beta}\right\}_{\beta<\alpha}$ satisfying (6). Put $H=\overline{\left[\left\{x_{i_{\beta}}\right\}_{\beta<\alpha}\right]}$ and $\nu=\sup _{\beta<\alpha}\left\{j_{\beta}\right\}$ (if $\alpha=1$, put $H=\{0\}$ and $\nu=1)$. By Lemma 3.1 there exist $\nu \leq \varrho<\gamma<\omega_{1}$ such that $x_{\varrho} \notin$ $\overline{\mathrm{co}}\left(H \cup\left\{ \pm N x_{i}\right\}_{\gamma \leq i<\omega_{1}}\right)$. So, we put $i_{\alpha}=\varrho, j_{\alpha}=\gamma$, and this completes the induction. Let $z_{\alpha}=x_{i_{\alpha}}$ for $\alpha<\omega_{1}$. By (6) we have $z_{\alpha} \notin \overline{\mathrm{co}}\left(\overline{\left[\left\{z_{\beta}: \beta<\alpha\right\}\right]} \cup\right.$ $\left.\left\{ \pm N z_{j}\right\}_{\alpha<j<\omega_{1}}\right)$. So, by the Hahn-Banach Theorem there exists $f_{\alpha} \in X^{*}$ such that

$$
1=f_{\alpha}\left(z_{\alpha}\right)>\sup \left\{f_{\alpha}(x): x \in \overline{\mathrm{co}}\left(\overline{\left[\left\{z_{\beta}: \beta<\alpha\right\}\right]} \cup\left\{ \pm N z_{j}\right\}_{\alpha<j<\omega_{1}}\right)\right\} .
$$

Clearly, $f_{\alpha}\left(z_{\beta}\right)=0$ if $\beta<\alpha$, and $\left|f_{\alpha}\left(N z_{\beta}\right)\right|<1$, i.e., $\left|f_{\alpha}\left(z_{\beta}\right)\right|<1 / N$, if $\alpha<\beta<\omega_{1}$. Finally, if we choose an uncountable subsequence $A \subset \omega_{1}$ with $\left\{\left\|f_{\alpha}\right\|: \alpha \in A\right\}$ bounded, then $\left\{\left(z_{\alpha}, f_{\alpha}\right): \alpha \in A\right\}$ is the UBABS of type $\eta$ we are looking for.

4. The Kunen-Shelah property $\mathrm{KS}_{4}$. A Banach space $X$ is said to have the Kunen-Shelah property $\mathrm{KS}_{4}$ if $X$ has no $\omega_{1}$-polyhedron. The implication $\mathrm{KS}_{4} \Rightarrow \mathrm{KS}_{3}$ was proved in [3]. It also follows from Proposition 3.2 and from Proposition 7.3 and a result of Sersouri [12].

Proposition 4.1. Let $Z$ be a Banach space and $X \subset Z$ a closed subspace such that $Z / X$ is separable. Then the following are equivalent:

(a) $Z \in \mathrm{KS}_{4}$.

(b) $X \in \mathrm{KS}_{4}$.

Proof. (a) $\Rightarrow(\mathrm{b})$. This is obvious.

(b) $\Rightarrow$ (a). Assume that $Z \notin \mathrm{KS}_{4}$; we will prove that $X \notin \mathrm{KS}_{4}$. By Proposition 2.2 there exists in $Z$ an UBABS $\left\{\left(z_{\alpha}, f_{\alpha}\right): \alpha<\omega_{1}\right\}$ of type $\eta \in[0,1)$ with $\left\|f_{\alpha}\right\| \leq M$ for all $\alpha<\omega_{1}$, for some $0<M<\omega_{1}$. Set $\varepsilon:=1-\eta$. Since $Z / X$ is separable, there exists an uncountable subset $I \subset \omega_{1}$ such that if $Q: Z \rightarrow Z / X$ is the canonical quotient mapping, then $\left\|Q z_{\alpha}-Q z_{\beta}\right\|<\varepsilon /(4 M)$ for every $\alpha, \beta \in I$. Fix $\tau \in I$ and define $y_{\alpha}=z_{\alpha}-z_{\tau}$ for $\alpha \in I$. Since $\left\|Q y_{\alpha}\right\|<\varepsilon /(4 M)$, there exists $x_{\alpha} \in X$ such that $\left\|x_{\alpha}-y_{\alpha}\right\|<\varepsilon /(4 M)$ for all $\alpha \in I$. Then for any $\alpha, \beta \in I, \alpha \neq \beta$, we have

$$
\begin{aligned}
f_{\alpha}\left(x_{\alpha}\right) & =f_{\alpha}\left(y_{\alpha}\right)+f_{\alpha}\left(x_{\alpha}-y_{\alpha}\right) \geq f_{\alpha}\left(y_{\alpha}\right)-M \frac{\varepsilon}{4 M}=f_{\alpha}\left(z_{\alpha}\right)-f_{\alpha}\left(z_{\tau}\right)-\frac{\varepsilon}{4} \\
& =1-f_{\alpha}\left(z_{\tau}\right)-\frac{\varepsilon}{4}>\eta-f_{\alpha}\left(z_{\tau}\right)+\frac{\varepsilon}{4} \geq f_{\alpha}\left(z_{\beta}\right)-f_{\alpha}\left(z_{\tau}\right)+\frac{\varepsilon}{4} \\
& =f_{\alpha}\left(y_{\beta}\right)+\frac{\varepsilon}{4}=f_{\alpha}\left(y_{\beta}\right)+M \frac{\varepsilon}{4 M} \geq f_{\alpha}\left(x_{\beta}\right),
\end{aligned}
$$

which implies that $\left\{x_{\alpha}: \alpha \in I\right\}$ is an uncountable polyhedron in $X$, i.e., $X \notin \mathrm{KS}_{4}$. 
In the following we obtain some characterizations of the property $\mathrm{KS}_{4}$. We first prove some lemmas.

Lemma 4.2. Let $X$ be a locally convex topological space, $\tau=\sigma\left(X, X^{*}\right)$, $f \in X^{*} \backslash\{0\}, C \subset f^{-1}(1)$ a bounded convex subset and $B=\operatorname{co}(C \cup(-C))$. Then $C$ is $\tau$-separable iff $B$ is $\tau$-separable.

Proof. Clearly, $B$ is $\tau$-separable whenever $C$ is. For the converse, suppose that $B$ is $\tau$-separable and choose a countable subset $A \subset C$ such that $D:=$ $\{t x-(1-t) y: x, y \in A, t \in[0,1]\}$ is $\tau$-dense in $B$. Now it is an easy exercise to prove that $C \subset \tau$-cl $(A)$, i.e., $C$ is $\tau$-separable.

Lemma 4.3. Let $X$ be a locally convex topological space, $\tau=\sigma\left(X, X^{*}\right)$, and $C \subset X$ a convex subset such that for some $f \in X^{*}$ there exists a countable subset $\mathcal{R} \subset \mathbb{R}$ satisfying:

(1) $\emptyset \neq(\inf \{f(x): x \in C\}, \sup \{f(x): x \in C\}) \subset \overline{\mathcal{R}}$.

(2) $C_{r}:=\{x \in C: f(x)=r\}$ is $\tau$-separable for each $r \in \mathcal{R}$.

Then $C$ is $\tau$-separable.

Proof. By hypothesis $\inf \{f(x): x \in C\}<\sup \{f(x): x \in C\}$. For each $r \in \mathcal{R}$, choose a countable subset $A_{r} \subset C_{r}$ such that $C_{r} \subset \tau-\operatorname{cl}\left(A_{r}\right)$. Let $A=\bigcup_{r \in \mathcal{R}} A_{r}$, a countable subset of $C$. We claim that $A$ is $\tau$-dense in $C$. Indeed, pick $z_{0} \in C$ arbitrarily and let $U$ be a $\tau$-neighborhood of $z_{0}$ in $C$. By hypothesis, there exists some $r \in \mathcal{R}$ such that $C_{r} \cap U \neq \emptyset$. So, $A_{r} \cap U \neq \emptyset$, whence $A \cap U \neq \emptyset$.

Proposition 4.4. Let $X$ be a Banach space. The following are equivalent:

(1) $X \in \mathrm{KS}_{4}$.

(2) $K \subset X^{*}$ is $w^{*}$-separable whenever $K$ is a $w^{*}$-compact convex symmetric subset such that $\|\cdot\|-\operatorname{int}(K) \neq \emptyset$.

(3) $K \subset X^{*}$ is $w^{*}$-separable whenever $K$ is a $w^{*}$-compact convex symmetric subset, i.e., $\sigma(X)=1=\tau(X)$.

(4) $K \subset X^{*}$ is $w^{*}$-separable whenever $K$ is a $w^{*}$-closed convex symmetric subset.

(5) $K \subset X^{*}$ is $w^{*}$-separable whenever $K$ is a $w^{*}$-closed convex subset.

Proof. (1) $\Rightarrow(2)$. This follows from Propositions 2.7 and 2.2, because if $K \subset X^{*}$ is a $w^{*}$-compact convex symmetric subset such that $\|\cdot\|-\operatorname{int}(K) \neq \emptyset$, then $K$ is the dual unit ball of $X^{*}$ when $X$ is equipped with the equivalent norm $|\cdot| \operatorname{such}$ that $|x|=\sup \left\{x^{*}(x): x^{*} \in K\right\}$ for every $x \in X$.

$(2) \Rightarrow(3)$. Let $K \subset X^{*}$ be a $w^{*}$-compact convex symmetric subset and set $K_{n}=K+\frac{1}{n} B\left(X^{*}\right)$, which is a $w^{*}$-compact convex symmetric subset of $X^{*}$ with nonempty interior. By (2) there is a countable family $\left\{x_{n, m}\right\}_{m \geq 1} \subset K_{n}$ 
such that $K_{n}={\overline{\left\{x_{n, m}: m \geq 1\right\}}}^{w^{*}}$ for every $n \geq 1$. Pick $k_{n, m} \in K$ such that $\left\|k_{n, m}-x_{n, m}\right\| \leq 1 / n$. Then it is easy to see that $K=\overline{\left\{k_{n, m}: n, m \geq 1\right\}} w^{*}$.

$(3) \Rightarrow(4)$. Let $K \subset X^{*}$ be a $w^{*}$-closed convex symmetric subset and define $K_{n}=K \cap n B\left(X^{*}\right)$. By (3), $K_{n}$ is $w^{*}$-separable and hence so is $K$, because $K=\bigcup_{n>1} K_{n}$.

$(4) \Rightarrow(5)$. It is enough to prove that if $K \subset X^{*}$ is a $w^{*}$-compact convex subset, then $K$ is $w^{*}$-separable. Without loss of generality, assume that $0 \notin K$. Let $f \in X$ be such that $0<\min \{f(k): k \in K\} \leq \max \{f(k)$ : $k \in K\}<\infty$. If $t \in[\min \{f(k): k \in K\}, \max \{f(k): k \in K\}]$, define $K_{t}=\{k \in K: f(k)=t\}$ and $C_{t}=\overline{\mathrm{co}} w^{*}\left(K_{t} \cup\left(-K_{t}\right)\right)$. By (4) and Lemma 4.2 each $C_{t}$ is $w^{*}$-separable. So, from Lemma 4.3 we conclude that $K$ is $w^{*}$ separable.

$(5) \Rightarrow(1)$. Suppose that there exists in $X$ a bounded $\omega_{1}$-polyhedron $\left\{x_{i}\right\}_{i<\omega_{1}}$. By Proposition 2.2, there exists in $X$ an UBABS $\left\{\left(x_{\alpha}, f_{\alpha}\right)\right\}_{\alpha<\omega_{1}} \subset$ $X \times X^{*}$ such that $\left\|f_{\alpha}\right\|=1,\left\|x_{\alpha}\right\| \leq M, f_{\alpha}\left(x_{\alpha}\right)=1$ and $f_{\alpha}\left(x_{\beta}\right) \leq 1-\varepsilon$ for every $\alpha, \beta<\omega_{1}, \alpha \neq \beta$, and some $1 \geq \varepsilon>0,1 \leq M<\infty$. Let $K=\overline{\mathrm{co}} w^{*}\left(\left\{f_{\alpha}: \alpha<\omega_{1}\right\}\right)$. Consider the $w^{*}$-open slices $U_{\alpha}=\{k \in K$ : $\left.k\left(x_{\alpha}\right)>1-\varepsilon / 3\right\}$ for all $\alpha<\omega_{1}$. Then $U_{\alpha}$ is a $w^{*}$-open neighborhood of $f_{\alpha}$ in $K$ and we can easily see that $U_{\alpha} \cap U_{\beta}=\emptyset$ whenever $\alpha \neq \beta$. Thus $K$ is $w^{*}$-nonseparable, a contradiction to (5). So, $X \in \mathrm{KS}_{4}$.

Question 3. Let $X$ be a Banach space. If $\tau(X)<1$, is $\tau(X)=0$ ? If $\tau(X)=0$, does $X$ have an uncountable $\omega$-independent family?

5. The Finet-Godefroy indices. If $X$ is a Banach space, the FinetGodefroy indices $d_{\infty}(X)$ and $\mu(X)$ were introduced in [1] and defined as follows:

$$
d_{\infty}(X)=\inf \left\{d(X, Y): Y \text { a subspace of } \ell_{\infty}(\mathbb{N})\right\},
$$

where $d(X, Y)$ is the Banach-Mazur distance. Clearly, $d_{\infty}(X)$ depends upon the norm $\|\cdot\|$ of $X$ and we see easily that: (i) $d_{\infty}(X) \in[1, \infty]$; (ii) $d_{\infty}(X)<$ $\infty$ iff $X$ is isomorphic to a subspace of $\ell_{\infty}(\mathbb{N})$; (iii) $d_{\infty}(X,\|\cdot\|)=1$ iff $(X,\|\cdot\|)$ is isometric to a subspace of $\ell_{\infty}(\mathbb{N})$ iff the dual unit ball $B\left(X^{*}\right)$ is $w^{*}$-separable. The corresponding isomorphic invariant index is

$$
\mu(X)=\sup \left\{d_{\infty}(X,|\cdot|)\right\},
$$

where the supremum is computed over the set of equivalent norms on $X$.

Proposition 5.1. Let $X$ be a Banach space. Then:

(1) $\mu(X)=\sigma(X)^{-1}\left(0^{-1}=\infty\right)$.

(2) If $X$ has an uncountable $\omega$-independent system, then $\mu(X)=\infty$.

Proof. (1) This follows from [1, Lemma III.1] and a simple calculation. (2) By Proposition 3.2 and 2.8 we find that $\sigma(X)=0$. Now apply (1). 
The following questions are proposed in [1]:

(1) It is clear that $\mu(X)=1$ if $X$ is separable. Is the converse true?

(2) Does there exist a nonseparable Banach space $X$ such that every quotient of $X$ is isometric to a subspace of $\ell_{\infty}(\mathbb{N})$ ?

In the following we answer these questions.

Proposition 5.2. Let $X$ be a Banach space. The following are equivalent:

(1) $X \in \mathrm{KS}_{4}$.

(2) Every quotient of $(X,|\cdot|)$ is isometric to a subspace of $\ell_{\infty}(\mathbb{N})$, for every equivalent norm $|\cdot|$ on $X$.

(3) $\mu(X)=1$.

(4) Every quotient of $X$ has the property $\mathrm{KS}_{4}$.

Proof. (1) $\Rightarrow(2)$. Let $|\cdot|$ be an equivalent norm on $X, Y \subset X$ a closed subspace and $Z=(X / Y,|\cdot|)$ the corresponding quotient space. Clearly, $\left(B\left(Z^{*}\right), w^{*}\right)=\left(B\left(Y^{\perp}\right), w^{*}\right)$. But $\left(B\left(Y^{\perp}\right), w^{*}\right)$ is $w^{*}$-separable by Proposition 4.4. So, $Z$ is isometric to a subspace of $\ell_{\infty}(\mathbb{N})$.

$(2) \Rightarrow(3)$. By $(2), d_{\infty}(X,|\cdot|)=1$ for every equivalent norm $|\cdot|$ on $X$. So, $\mu(X)=1$.

$(3) \Rightarrow(4)$. Since $\mu(X / Y) \leq \mu(X)$ for every quotient $X / Y$ (see $[1$, Th. III-2]), (3) implies that $\mu(X / Y)=1$, i.e., $\sigma(X / Y)=1$. So, by Proposition 4.4 we infer that $X / Y \in \mathrm{KS}_{4}$.

$(4) \Rightarrow(1)$. This is obvious.

Corollary 5.3. If $X$ is either the space $C(K)$, under $C H$ and $K$ being the Kunen compact space, or the space $S$ of Shelah, under $\diamond_{\aleph_{1}}$, then $X$ is nonseparable, $\mu(X)=1$ and every quotient of $(X,|\cdot|)$ is isometric to a subspace of $\ell_{\infty}(\mathbb{N})$, for every equivalent norm $|\cdot|$ of $X$.

Proof. This follows from Proposition 5.2 since in both cases $X \in \mathrm{KS}_{4}$ (see Section 6).

REMARKs. (1) The fact that every quotient of $(X,|\cdot|)$ is isometric to a subspace of $\ell_{\infty}(\mathbb{N})$ for every equivalent norm $|\cdot|$ of $X$, when $X=C(K), K$ being the Kunen compact, was shown in [4, Cor. 4.5].

(2) In [1] it is asked if $\mu(X)=\infty$ whenever the Banach space $X$ satisfies $\mu(X)>1$. In fact, no Banach space $X$ with $1<\mu(X)<\infty$ is known. Observe that $1<\mu(X)<\infty$ implies that $X \in \mathrm{KS}_{3}$ but $X \notin \mathrm{KS}_{4}$, because: (i) $1<\mu(X)<\infty$ iff $1>\sigma(X)>0$ by Proposition 5.1; (ii) $1>\sigma(X)$ iff $X \notin \mathrm{KS}_{4}$ by Proposition 4.4; and (iii) $\sigma(X)>0$ implies $X \in \mathrm{KS}_{3}$ by Propositions 3.2 and 2.8 . 
6. The Kunen-Shelah property $\mathrm{KS}_{5}$. Let $\theta$ be an ordinal. A convex right-separated $\theta$-family in a Banach space $X$ is a bounded family $\left\{x_{i}\right\}_{i<\theta} \subset$ $X$ such that $x_{j} \notin \overline{\mathrm{co}}\left(\left\{x_{i}: j<i<\theta\right\}\right)$ for every $j \in \theta$. A family $\left\{C_{\alpha}\right\}_{\alpha<\theta}$ of convex closed bounded subsets in $X$ is said to be a contractive (resp. expansive) $\theta$-onion iff $C_{\alpha} \subsetneq C_{\beta}$ (resp. $C_{\beta} \subsetneq C_{\alpha}$ ) whenever $\beta<\alpha<\theta$. It is easy to prove that $X$ has a contractive $\theta$-onion iff $X$ has a convex rightseparated $\theta$-family. In the dual Banach space $X^{*}$ one can define a contractive (resp. expansive) $w^{*}-\theta$-onion in an analogous way, using the $w^{*}$-topology instead of the w-topology.

A Banach space $X$ is said to have the Kunen-Shelah property $\mathrm{KS}_{5}$ if $X$ has no contractive uncountable onion. If $X$ has a $\tau$-polyhedron $\left\{x_{\alpha}: \alpha<\tau\right\}$, it is clear that $\left\{C_{\alpha}: \alpha<\tau\right\}$, where $C_{\alpha}=\overline{\mathrm{co}}\left(\left\{x_{\beta}: \alpha<\beta<\tau\right\}\right)$, is a contractive $\tau$-onion. So, the property $\mathrm{KS}_{5}$ implies $\mathrm{KS}_{4}$, whence by Proposition 3.2 we get $\mathrm{KS}_{5} \Rightarrow \mathrm{KS}_{3}$, a result proved by Sersouri in [12].

Proposition 6.1. Let $X$ be a Banach space. Then:

(1) $X$ has a contractive $\omega_{1}$-onion iff $X^{*}$ has an expansive $w^{*}-\omega_{1}$-onion.

(2) $X$ has an expansive $\omega_{1}$-onion iff $X^{*}$ has a contractive $w^{*}$ - $\omega_{1}$-onion.

(3) $X$ is nonseparable iff $X^{*}$ has a contractive $w^{*}-\omega_{1}$-onion.

Proof. (1) Assume that $X$ has a contractive $\omega_{1}$-onion, i.e., there exists a sequence $\left\{x_{\alpha}\right\}_{\alpha<\omega_{1}} \subset B(X)$ such that $x_{\alpha} \notin \overline{\mathrm{co}}\left(\left\{x_{\beta}\right\}_{\alpha<\beta<\omega_{1}}\right)$. By the HahnBanach Theorem there exists $f_{\alpha} \in X^{*}$ such that

$$
f_{\alpha}\left(x_{\alpha}\right)>\sup \left\{f_{\alpha}\left(x_{\beta}\right): \alpha<\beta<\omega_{1}\right\}=: e_{\alpha} .
$$

By passing to a subsequence, we can suppose that there exist $0<\varepsilon, M<\infty$ and $r \in \mathbb{R}$ such that $\left\|f_{\alpha}\right\| \leq M, f_{\alpha}\left(x_{\alpha}\right)-e_{\alpha} \geq \varepsilon>0$ and $\left|r-f_{\alpha}\left(x_{\alpha}\right)\right| \leq \varepsilon / 4$ for all $\alpha<\omega_{1}$. Hence, if $\beta<\alpha<\omega_{1}$, we have

$$
f_{\alpha}\left(x_{\alpha}\right) \geq r-\varepsilon / 4>r-3 \varepsilon / 4 \geq f_{\beta}\left(x_{\beta}\right)-\varepsilon \geq e_{\beta} \geq f_{\beta}\left(x_{\alpha}\right),
$$

which implies that $f_{\alpha} \notin \overline{\mathrm{CO}}^{w^{*}}\left(\left\{f_{\beta}: \beta<\alpha\right\}\right)=$ : $K_{\alpha}$, i.e., $\left\{K_{\alpha}: \alpha<\omega_{1}\right\}$ is an expansive $w^{*}-\omega_{1}$-onion in $X^{*}$.

The converse implication is analogous.

(2) Use the same argument as in (1).

(3) Apply (2) and the fact that $X$ has an expansive $\omega_{1}$-onion iff $X$ is nonseparable.

A Banach space has the property $\mathrm{HL}(1)$ (for short, $X \in \mathrm{HL}(1)$ ) whenever for every family $\left\{U_{i}\right\}_{i \in I}$ of open semi-spaces of $X$ there exists a countable subset $\left\{i_{n}\right\}_{n \geq 1} \subset I$ such that $\bigcup_{n \geq 1} U_{i_{n}}=\bigcup_{i \in I} U_{i}$, i.e., every closed convex subset of $X$ is the intersection of a countable family of closed semi-spaces of $X$. 
Proposition 6.2. Let $X$ be a Banach space. Then the following are equivalent:

(1) $X \in \mathrm{KS}_{5}$.

(2) Every convex subset of $X^{*}$ is $w^{*}$-separable.

(3) $X \in \mathrm{HL}(1)$.

Proof. (1) $\Leftrightarrow(2)$. By Proposition 6.1, $X$ has no contractive uncountable onion iff $X^{*}$ has no expansive uncountable $w^{*}$-onion, and it is trivial to prove that this occurs iff every convex subset of $X^{*}$ is $w^{*}$-separable.

$(2) \Rightarrow(3)$. Suppose that $X \notin \mathrm{HL}(1)$ and let $\mathfrak{F}=\left\{U_{i}\right\}_{i<\omega_{1}}$ be an uncountable family of open semi-spaces of $X$ such that $\mathfrak{F}$ has no countable subcover. Assume that $U_{i}=\left\{x \in X: x_{i}^{*}(x)<a_{i}\right\}$ with $a_{i} \neq 0$ for all $i<\omega_{1}$ (if $a_{i}=0$ for some $i<\omega_{1}$, we replace $U_{i}$ by the family $U_{i n}=\left\{x \in X: x_{i}^{*}(x)<\right.$ $-1 / n\}, n \geq 1)$. Dividing by $\left|a_{i}\right|$, we can suppose that each $U_{i}$ has the expression $U_{i}=\left\{x \in X: y_{i}^{*}(x)<\varepsilon_{i}\right\}$ with $\varepsilon_{i}= \pm 1$ and $y_{i}^{*}=x_{i}^{*} /\left|a_{i}\right|$. Set $\mathfrak{F}_{1}=\left\{U_{i} \in \mathfrak{F}: \varepsilon_{i}=+1\right\}$ and $\mathfrak{F}_{2}=\left\{U_{i} \in \mathfrak{F}: \varepsilon_{i}=-1\right\}$. It is clear that either $\mathfrak{F}_{1}$ or $\mathfrak{F}_{2}$ has no countable subcover.

Assume that $\mathfrak{F}_{1}$ does not admit a countable subcover (the argument for $\mathfrak{F}_{2}$ is similar). So, there exists an uncountable family $\left\{V_{\alpha}: \alpha<\omega_{1}\right\} \subset \mathfrak{F}_{1}$, $V_{\alpha}=\left\{x \in X: z_{\alpha}^{*}(x)<1\right\}$, such that there exist $x_{\alpha} \in V_{\alpha} \backslash \bigcup_{\beta<\alpha} V_{\beta}$ for each $\alpha<\omega_{1}$. Put $A=\operatorname{co}\left\{z_{i}^{*}\right\}_{i<\omega_{1}}$, which is $w^{*}$-separable by hypothesis. Thus, we can find $\varrho<\omega_{1}$ such that $A \subset \overline{\mathrm{co}} w^{*}\left(\left\{z_{i}^{*}\right\}_{i \leq \varrho}\right)$. Pick $\varrho<\alpha<\omega_{1}$. As $x_{\alpha} \in V_{\alpha} \backslash \bigcup_{\beta<\alpha} V_{\beta}$, we see that $z_{\alpha}^{*}\left(x_{\alpha}\right)<1$ and $z_{\beta}^{*}\left(x_{\alpha}\right) \geq 1$ for every $\beta<\alpha$. Let $C=\left\{x^{*} \in X^{*}: x^{*}\left(x_{\alpha}\right) \geq 1\right\}$, which is a convex $w^{*}$-closed subset of $X^{*}$. Since $z_{i}^{*} \in C$ for all $i \leq \varrho$, it follows that $A \subset C$. So, $z_{\alpha}^{*} \notin C$ and $z_{\alpha}^{*} \in A$, a contradiction which proves (3).

$(3) \Rightarrow(1)$. Suppose that $X$ has a contractive $\omega_{1}$-onion $\left\{C_{\alpha}\right\}_{\alpha<\omega_{1}}$. We choose vectors $x_{\alpha} \in C_{\alpha} \backslash C_{\alpha+1}$ and a sequence $\left\{U_{\alpha}\right\}_{\alpha<\omega_{1}}$ of open semi-spaces such that $x_{\alpha} \in U_{\alpha}$ and $U_{\alpha} \cap C_{\alpha+1}=\emptyset$. Clearly, no countable subfamily of $\left\{U_{\alpha}\right\}_{\alpha<\omega_{1}}$ covers $\left\{x_{\alpha}\right\}_{\alpha<\omega_{1}}$, which contradicts (3).

Remark. If $X$ is a Banach space, we write $X \in \mathrm{L}(1)$ if from every cover of $X$ by open semi-spaces we can choose a countable subcover. Clearly, $X$ has the property (C) of Corson iff $X \in \mathrm{L}(1)$. Since $X \in \mathrm{HL}(1) \Rightarrow X \in \mathrm{L}(1)$, we find that $X \in \mathrm{KS}_{5}$ implies $X \in(\mathrm{C})$.

Proposition 6.3. If $X$ is either the space $C(K)$, under $C H$ and $K$ being the Kunen compact space, or the space $S$ of Shelah, under $\diamond_{\aleph_{1}}$, then $X \in \mathrm{KS}_{5}$.

Proof. The space $C(K), K$ being the Kunen compact space, satisfies $C(K) \in \mathrm{KS}_{5}$ because for every uncountable family $\left\{x_{i}: i \in I\right\} \subset C(K)$, there exists $j \in I$ such that $x_{j} \in \operatorname{wcl}\left(\left\{x_{i}: i \in I \backslash\{j\}\right\}\right)$ (wcl = weak closure). It is clear that a space with this property cannot have an $\omega_{1}$-onion. 
The space $S$ of Shelah has the property (see [13, Lemma 5.2]) that if $\left\{y_{i}\right\}_{i<\omega_{1}} \subset S$ is an uncountable sequence, then for every $\varepsilon>0$ and $n \geq 1$, there exist $i_{0}<i_{1}<\ldots<i_{n}<\omega_{1}$ such that

$$
\left\|y_{i_{0}}-\frac{1}{n}\left(y_{i_{1}}+\ldots+y_{i_{n}}\right)\right\| \leq \frac{1}{n}\left\|y_{i_{0}}\right\|+\varepsilon .
$$

Assume that $S$ has an $\omega_{1}$-onion $\left\{C_{\alpha}: 1 \leq \alpha<\omega_{1}\right\}$, with $C_{1} \subset B(S)$. Choose $x_{\alpha} \in C_{\alpha} \backslash C_{\alpha+1}$ and let $\eta_{\alpha}:=\operatorname{dist}\left(x_{\alpha}, C_{\alpha+1}\right)$, which satisfies $\eta_{\alpha}>0$. By passing to a subsequence, it can be assumed that $\eta_{\alpha} \geq \eta>0$ for all $\alpha<\omega_{1}$. Let $m \in \mathbb{N}$ satisfy $1 / m<\eta / 2$. By (7) there exist $i_{0}<i_{1}<\ldots<i_{m}<\omega_{1}$ such that

$$
\left\|x_{i_{0}}-\frac{1}{m}\left(x_{i_{1}}+\ldots+x_{i_{m}}\right)\right\| \leq \frac{1}{m}\left\|x_{i_{0}}\right\|+\frac{\eta}{2}<\eta .
$$

Since $\frac{1}{m}\left(x_{i_{1}}+\ldots+x_{i_{m}}\right) \in C_{i_{0}+1}$ and $\operatorname{dist}\left(x_{i_{0}}, C_{i_{0}+1}\right) \geq \eta$, we get a contradiction which proves that $S \in \mathrm{KS}_{5}$.

7. $\mathrm{KS}_{4}$ and $\mathrm{KS}_{5}$ are equivalent. If $X$ is Asplund or has the property (C) of Corson, it is easy to prove that $X \in \mathrm{KS}_{4} \Leftrightarrow X \in \mathrm{KS}_{5}$. In the following we prove the equivalence $\mathrm{KS}_{5} \Leftrightarrow \mathrm{KS}_{4}$ in general. A sequence $\left\{C_{\alpha}\right.$ : $\left.\alpha<\omega_{1}\right\}$ of convex closed bounded subsets of a Banach space $X$ is said to be a generalized $\omega_{1}$-onion if $\emptyset \neq C_{\alpha} \subset C_{\beta}$ for $\beta<\alpha$, and there exists a subsequence $\left\{\alpha_{\beta}\right\}_{\beta<\omega_{1}} \subset \omega_{1}$, with $\alpha_{\beta_{1}}<\alpha_{\beta_{2}}$ if $\beta_{1}<\beta_{2}$, such that $C_{\alpha_{\beta_{1}}} \neq$ $C_{\alpha_{\beta_{2}}}$, i.e., $\left\{C_{\alpha_{\beta}}: \beta<\omega_{1}\right\}$ is an $\omega_{1}$-onion. For $C \subset X$, denote by $\operatorname{cone}(C)$ the closed convex cone generated by $C$. Observe that if $C$ is convex, then $\operatorname{cone}(C)=\operatorname{cl}\left(\bigcup_{\lambda \geq 0} \lambda C\right)$.

LEMma 7.1. Let $X$ be a Banach space, $C \subset X$ a convex closed separable subset and $\left\{C_{\alpha}: 1 \leq \alpha<\omega_{1}\right\}$ a generalized $\omega_{1}$-onion in $X$.

(1) If $\operatorname{dist}\left(C, C_{\alpha}\right)=0$ for every $\alpha<\omega_{1}$, then for every $\varepsilon>0$ there exists $c_{\varepsilon} \in C$ such that $\operatorname{dist}\left(c_{\varepsilon}, C_{\alpha}\right) \leq \varepsilon$ for every $\alpha<\omega_{1}$.

(2) There are two mutually exclusive alternatives: either

(A) there exist two ordinals $\beta<\alpha<\omega_{1}$ and $z \in C_{\beta}$ such that $z \notin \overline{\mathrm{co}}\left([C] \cup \operatorname{cone}\left(C_{\alpha}\right)\right)$ or

(B) for every pair of ordinals $\beta<\alpha<\omega_{1}$ we have $C_{\beta} \subset \overline{\mathrm{co}}([C] \cup$ cone $\left.\left(C_{\alpha}\right)\right)$. In this case,

$$
\overline{\operatorname{co}}\left([C] \cup \operatorname{cone}\left(C_{\alpha}\right)\right)=\overline{\operatorname{co}}\left([C] \cup \operatorname{cone}\left(C_{\beta}\right)\right), \quad \forall \alpha, \beta<\omega_{1},
$$

and for every $\varepsilon>0$ there exists $c_{\varepsilon} \in X$ such that $\operatorname{dist}\left(c_{\varepsilon}, C_{\alpha}\right) \leq \varepsilon$ for every $\alpha<\omega_{1}$. 
Proof. (1) For every $\alpha<\omega_{1}$ and $n \geq 1$ consider $C(\alpha, n)=\{x \in C$ : $\left.\operatorname{dist}\left(x, C_{\alpha}\right) \leq 1 / n\right\}$. Then $\left\{C(\alpha, n): \alpha<\omega_{1}\right\}$ is a family of nonempty closed convex subsets such that $C(\alpha, n) \supset C(\beta, n)$ if $\alpha<\beta$, with the countable intersection property. Since $C$ is separable, we conclude that $\bigcap_{\alpha<\omega_{1}} C(\alpha, n) \neq \emptyset$ for every $n \geq 1$. So, if for every $n \geq 1$ we pick $c_{n} \in$ $\bigcap_{\alpha<\omega_{1}} C(\alpha, n)$, then $\operatorname{dist}\left(c_{n}, C_{\alpha}\right) \leq 1 / n$ for every $\alpha<\omega_{1}$.

(2) Clearly, the alternatives (A) and (B) are mutually exclusive. Suppose that (B) holds. Since $[C]$ is separable there exist two ordinals $\beta_{0}<\alpha_{0}<\omega_{1}$ and $z_{0} \in C_{\beta_{0}} \backslash C_{\alpha_{0}}$ such that $z_{0} \notin \overline{[C]}$ but $z_{0} \in \overline{\mathrm{co}}\left([C] \cup \operatorname{cone}\left(C_{\alpha}\right)\right)$ for every $\alpha<\omega_{1}$.

Claim. If $H=\overline{\left[C \cup\left\{z_{0}\right\}\right]}$, then $\operatorname{dist}\left(H, C_{\alpha}\right)=0$ for every $\alpha<\omega_{1}$.

Indeed, let $\varepsilon_{0}=\operatorname{dist}\left(z_{0}, \overline{[C]}\right)$ and $n_{0} \geq 1$ be such that $2 / n_{0}<\varepsilon_{0}$. Observe that for every $\alpha<\omega_{1}$ and $\varepsilon>0$ we can choose $\lambda \in[0,1), \mu>0, w \in[C]$ and $v \in C_{\alpha}$ such that

$$
\left\|\lambda w+(1-\lambda) \mu v-z_{0}\right\| \leq \varepsilon .
$$

Let $M>0$ be such that $C_{1} \subset B(0, M)$. We claim that if we pick $\alpha<\omega_{1}$, $n \geq n_{0}, \lambda \in[0,1), \mu>0, w \in[C]$ and $v \in C_{\alpha}$ satisfying (8) with $\varepsilon=1 / n$, then $(1-\lambda) \mu \geq 1 /\left(n_{0} M\right)$. Indeed, otherwise

$$
\begin{aligned}
\varepsilon_{0} & \leq\left\|\lambda w-z_{0}\right\|=\left\|\lambda w+(1-\lambda) \mu v-z_{0}-(1-\lambda) \mu v\right\| \\
& \leq\left\|\lambda w+(1-\lambda) \mu v-z_{0}\right\|+\|(1-\lambda) \mu v\| \\
& \leq \frac{1}{n_{0}}+\frac{1}{n_{0}}<\varepsilon_{0},
\end{aligned}
$$

which is a contradiction. So, for every $\alpha, n, \lambda, \mu, w$ and $v$ as above we have

$$
\left\|\frac{z_{0}}{(1-\lambda) \mu}-\frac{\lambda}{(1-\lambda) \mu} w-v\right\| \leq \frac{1}{(1-\lambda) \mu n} \leq \frac{n_{0} M}{n},
$$

and this proves that $\operatorname{dist}\left(H, C_{\alpha}\right)=0$ for every $\alpha<\omega_{1}$.

As $H$ is separable, given $\varepsilon>0$, applying (1) we can choose $c_{\varepsilon} \in X$ such that $\operatorname{dist}\left(c_{\varepsilon}, C_{\alpha}\right) \leq \varepsilon$ for every $\alpha<\omega_{1}$, and this completes the proof.

Proposition 7.2. Let $X$ be a Banach space without the property $(\mathrm{C})$ of Corson. Then there exists a sequence $\left\{\left(y_{\alpha}, y_{\alpha}^{*}\right): \alpha<\omega_{1}\right\} \subset X \times X^{*}$ such that $y_{\alpha}^{*}\left(y_{\alpha}\right)=1$ for all $\alpha<\omega_{1}$ but $y_{\alpha}^{*}\left(y_{\beta}\right)=0$ if $\beta<\alpha$, and $y_{\alpha}^{*}\left(y_{\beta}\right) \leq 0$ if $\beta>\alpha$. So, $X$ has an $\omega_{1}$-polyhedron and $X \notin \mathrm{KS}_{4}$.

Proof. Since $X$ fails (C), it is easy to see that there exists in $X$ an $\omega_{1}$ onion $\left\{C_{\alpha}: \alpha<\omega_{1}\right\}$ such that $\bigcap_{\alpha<\omega_{1}} C_{\alpha}=\emptyset$. Using transfinite induction with $\omega_{1}$ steps we construct: 
(1) A sequence $\left\{n_{\alpha}: \alpha<\omega_{1}\right\} \subset\{0,1\}$ such that if $p(\alpha)=\mid\{\beta \leq \alpha$ : $\left.n_{\beta}=1\right\} \mid$ then $p(\alpha)<\aleph_{0}$.

(2) Two sequences $\left\{\varrho_{\gamma}, \tau_{\gamma}: \gamma<\omega_{1}\right\}$ of ordinals such that $1 \leq \varrho_{\gamma}<\tau_{\gamma} \leq$ $\varrho_{\beta}<\omega_{1}$ if $\gamma<\beta<\omega_{1}$.

(3) For each $\alpha<\omega_{1}$, a generalized $\omega_{1}$-onion $\left\{C_{\beta}^{(\alpha)}: \varrho_{\alpha} \leq \beta<\omega_{1}\right\}$ such that $C_{\gamma} \supset C_{\gamma}^{(\alpha)} \supset C_{\gamma}^{(\beta)} \neq \emptyset$ if $\alpha \leq \beta<\omega_{1}$ and $\varrho_{\beta} \leq \gamma<\omega_{1}$.

(4) For each $\alpha$ with $n_{\alpha}=0$, an element $y_{\alpha} \in C_{\varrho_{\alpha}}^{(\alpha)}$ such that if $H_{\alpha}=$ $\overline{\left[\left\{y_{\beta}: \beta<\alpha, n_{\beta}=0\right\}\right]}$ then $y_{\alpha} \notin \overline{\mathrm{co}}\left(H_{\alpha} \cup \operatorname{cone}\left(C_{\tau_{\alpha}}^{(\alpha)}\right)\right)$. Also, in this case we demand that $C_{\gamma}^{(\alpha)}=\bigcap_{\beta<\alpha} C_{\gamma}^{(\beta)}$ for every $\varrho_{\alpha} \leq \gamma<\omega_{1}$.

(5) For each $\alpha$ with $n_{\alpha}=1$, a vector $a_{p(\alpha)} \in X$ such that $C_{\beta}^{(\alpha)} \subset$ $B\left(a_{p(\alpha)}, 2^{-p(\alpha)}\right)$ for every $\tau_{\alpha} \leq \beta<\omega_{1}$, which will imply that

$\operatorname{diam}\left(C_{\beta}^{(\alpha)}\right) \leq 2^{-p(\alpha)+1}, \quad \operatorname{dist}\left(a_{p(\alpha)}, C_{\beta}^{(\alpha)}\right) \leq 2^{-p(\alpha)}, \quad \forall \tau_{\alpha} \leq \beta<\omega_{1}$.

STEP 1 . We choose $n_{1}=0, \varrho_{1}=1, \tau_{1}=2, C_{\beta}^{(1)}=C_{\beta}$ for every $1 \leq \beta<$ $\omega_{1}, y_{1} \in C_{1} \backslash C_{2}$ arbitrary and $H_{1}=\{0\}$.

SteP $\alpha+1<\omega_{1}$. Suppose all the steps $\beta \leq \alpha$ satisfying the above requirements are constructed. By hypothesis $\left\{C_{\beta}^{(\alpha)}: \tau_{\alpha} \leq \beta<\omega_{1}\right\}$ is a generalized $\omega_{1}$-onion. By Lemma 7.1 there are two mutually exclusive alternatives:

(A) There exist two ordinals $\tau_{\alpha} \leq \beta_{0}<\alpha_{0}<\omega_{1}$ and a vector $z_{0} \in C_{\beta_{0}}^{(\alpha)}$ such that $z_{0} \notin \overline{\mathrm{co}}\left(H_{\alpha} \cup \operatorname{cone}\left(C_{\alpha_{0}}^{(\alpha)}\right)\right)$. Then we set $\varrho_{\alpha+1}=\beta_{0}, \tau_{\alpha+1}=\alpha_{0}$, $n_{\alpha+1}=0, y_{\alpha+1}=z_{0}$ and $C_{\beta}^{(\alpha+1)}=C_{\beta}^{(\alpha)}$ for every $\varrho_{\alpha+1} \leq \beta<\omega_{1}$.

(B) If (A) does not hold, there exists $c \in X$ such that $\operatorname{dist}\left(c, C_{\beta}^{(\alpha)}\right) \leq$ $2^{-(p(\alpha)+2)}$ for every $\tau_{\alpha} \leq \beta<\omega_{1}$. In this case we set $n_{\alpha+1}=1, p(\alpha+1)$ $=p(\alpha)+1, \varrho_{\alpha+1}=\tau_{\alpha}, \tau_{\alpha+1}=\tau_{\alpha}+1, a_{p(\alpha+1)}=c$ and $C_{\beta}^{(\alpha+1)}=$ $B\left(a_{p(\alpha+1)}, 2^{-p(\alpha+1)}\right) \cap C_{\beta}^{(\alpha)}$ for every $\varrho_{\alpha+1} \leq \beta<\omega_{1}$. Since $n_{\alpha+1}=1$ we do not choose $y_{\alpha+1}$.

STEP $\alpha<\omega_{1}, \alpha$ a limit ordinal. Let $\alpha<\omega_{1}$ be a limit ordinal, and suppose all the steps $\beta<\alpha$ satisfying the above requirements are constructed.

Claim. $\left|\left\{\beta<\alpha: n_{\beta}=1\right\}\right|<\aleph_{0}$.

Indeed, otherwise we would have a sequence of ordinals $\left\{\beta_{m}\right\}_{m \geq 1} \uparrow \alpha$, with $\beta_{m}<\beta_{m+1}<\alpha$, such that $n_{\beta_{m}}=1$ for every $m \geq 1$. Obviously $p\left(\beta_{m}\right) \uparrow+\infty$ as $m \rightarrow \infty$. The sequence $\left\{a_{p\left(\beta_{m}\right)}\right\}_{m \geq 1}$ is a Cauchy sequence. Indeed, if $r<s$ are two integers, then for every $\tau_{\beta_{s}} \leq \beta<\omega_{1}$, since $C_{\beta}^{\left(\beta_{s}\right)} \subset$ $C_{\beta}^{\left(\beta_{r}\right)}$, we have 


$$
\begin{aligned}
\operatorname{dist}\left(a_{p\left(\beta_{r}\right)}, a_{p\left(\beta_{s}\right)}\right) & \leq \operatorname{dist}\left(a_{p\left(\beta_{r}\right)}, C_{\beta}^{\left(\beta_{r}\right)}\right)+\operatorname{diam}\left(C_{\beta}^{\left(\beta_{r}\right)}\right)+\operatorname{dist}\left(a_{p\left(\beta_{s}\right)}, C_{\beta}^{\left(\beta_{r}\right)}\right) \\
& \leq 2^{-p\left(\beta_{r}\right)}+2^{-p\left(\beta_{r}\right)+1}+2^{-p\left(\beta_{s}\right) \stackrel{r, s \rightarrow \infty}{\longrightarrow} 0 .}
\end{aligned}
$$

Let $a_{0}:=\lim _{m \rightarrow \infty} a_{p\left(\beta_{m}\right)}$ and $\gamma_{0}=\sup \left\{\tau_{\beta}: \beta<\alpha\right\}$. Then $a_{0} \in C_{\gamma}$ for every $\gamma_{0} \leq \gamma<\omega_{1}$ because

$$
\operatorname{dist}\left(a_{0}, C_{\gamma}\right) \leq \operatorname{dist}\left(a_{0}, a_{p\left(\beta_{m}\right)}\right)+\operatorname{dist}\left(a_{p\left(\beta_{m}\right)}, C_{\gamma}^{\left(\beta_{m}\right)}\right) \stackrel{m \rightarrow \infty}{\longrightarrow} 0 .
$$

Hence $\bigcap_{\alpha<\omega_{1}} C_{\alpha} \neq \emptyset$, a contradiction which proves the Claim.

Define as above $\gamma_{0}=\sup \left\{\tau_{\beta}: \beta<\alpha\right\}$ and let $D_{\gamma}:=\bigcap_{\beta<\alpha} C_{\gamma}^{(\beta)}$ for every $\gamma_{0} \leq \gamma<\omega_{1}$. By the Claim and the construction of the previous steps we have:

(a) There exists an ordinal $\delta_{0}<\alpha$ such that $n_{\delta}=0$ for every $\delta_{0} \leq \delta<\alpha$. So, $p(\delta)=p\left(\delta_{0}\right)$ for every $\delta \in\left[\delta_{0}, \alpha\right)$.

(b) For every $\gamma_{0} \leq \gamma<\omega_{1}$ we have $D_{\gamma}=C_{\gamma}^{\left(\delta_{0}\right)}$, which by the induction hypothesis implies that $\left\{D_{\gamma}: \gamma_{0} \leq \gamma<\omega_{1}\right\}$ is a generalized $\omega_{1}$-onion.

If $H_{\alpha}:=\overline{\left[\left\{y_{\beta}: \beta<\alpha, n_{\beta}=0\right\}\right]}$, by Lemma 7.1 we have the following mutually exclusive alternatives:

(A) There are two ordinals $\gamma_{0} \leq \beta_{0}<\alpha_{0}<\omega_{1}$ and a vector $z_{0} \in D_{\beta_{0}}$ such that $z_{0} \notin \overline{\operatorname{co}}\left(H_{\alpha} \cup \operatorname{cone}\left(D_{\alpha_{0}}\right)\right)$. In this case we set $\varrho_{\alpha}=\beta_{0}, \tau_{\alpha}=\alpha_{0}$, $n_{\alpha}=0, y_{\alpha}=z_{0}$ and $C_{\beta}^{(\alpha)}=D_{\beta}$ for every $\varrho_{\alpha} \leq \beta<\omega_{1}$.

(B) If (A) does not hold, there exists $c \in X \operatorname{such}$ that $\operatorname{dist}\left(c, D_{\gamma}\right) \leq$ $2^{-p\left(\delta_{0}\right)+2}$ for every $\gamma_{0} \leq \gamma<\omega_{1}$. In this case we set $n_{\alpha}=1, p(\alpha)=$ $p\left(\delta_{0}\right)+1, \varrho_{\alpha}=\gamma_{0}, \tau_{\alpha}=\varrho_{\alpha}+1, a_{p(\alpha)}=c$ and $C_{\gamma}^{(\alpha)}=B\left(a_{p(\alpha)}, 2^{-p(\alpha)}\right) \cap D_{\gamma}$ for $\gamma_{0} \leq \gamma<\omega_{1}$. Since $n_{\alpha}=1$ we do not choose $y_{\alpha}$.

This completes the induction.

Obviously, there exists $\varrho<\omega_{1}$ such that $n_{\alpha}=0$ for every $\varrho \leq \alpha<\omega_{1}$, which gives us the sequence $\left\{y_{\alpha}: \varrho \leq \alpha<\omega_{1}\right\}$ such that

$$
y_{\alpha} \notin \overline{\mathrm{co}}\left(\overline{\left[\left\{y_{\beta}: \varrho \leq \beta<\alpha\right\}\right]} \cup \operatorname{cone}\left(\left\{y_{\beta}: \alpha<\beta<\omega_{1}\right\}\right)\right)=: K_{\alpha}
$$

for every $\varrho \leq \alpha<\omega_{1}$. Therefore, by the Hahn-Banach Theorem there exists $y_{\alpha}^{*} \in X^{*}$ such that $y_{\alpha}^{*}\left(y_{\alpha}\right)=1$ but $\sup \left\{y_{\alpha}^{*}(y): y \in K_{\alpha}\right\}<1$. In particular, $y_{\alpha}^{*}\left(y_{\beta}\right)=0$ if $\varrho \leq \beta<\alpha$, and $y_{\alpha}^{*}\left(y_{\beta}\right) \leq 0$ if $\alpha<\beta<\omega_{1}$.

Proposition 7.3. Let $X$ be a Banach space. We have:

(1) If $X \in \mathrm{KS}_{4}$, then $X \in(\mathrm{C})$.

(2) $X \in \mathrm{KS}_{4}$ iff $X \in \mathrm{KS}_{5}$.

Proof. (1) This follows from Proposition 7.2 where it is proved that if $X \notin(\mathrm{C})$ then $X$ has an $\omega_{1}$-polyhedron. 
(2) Clearly, $X \in \mathrm{KS}_{5}$ implies $X \in \mathrm{KS}_{4}$. Assume that $X \in \mathrm{KS}_{4}$. By (1) we see that $X \in(\mathrm{C})$. In order to prove that $X \in \mathrm{KS}_{5}$, by Proposition 6.2 it is enough to prove that every convex subset $C \subset X^{*}$ is $w^{*}$-separable. Since $X \in \mathrm{KS}_{4}, \bar{C}^{w^{*}}$ is $w^{*}$-separable by Proposition 4.4. So, there exists a countable family $\left\{z_{n}: n \geq 1\right\} \subset \bar{C}^{w^{*}} w^{*}$-dense in $\bar{C}^{w^{*}}$. Since $X \in(\mathrm{C})$, by $[10$, p. 147$]$ there exists a countable family $\left\{z_{n m}: n, m \geq 1\right\} \subset C$ such that $z_{n} \in \overline{\mathrm{CO}}^{w^{*}}\left(\left\{z_{n m}: m \geq 1\right\}\right)$ for every $n \geq 1$. So, $C$ is $w^{*}$-separable.

Remarks. A nonseparable Banach space $X$ has the Kunen-Shelah property $\mathrm{KS}_{6}$ if for every uncountable family $\left\{x_{i}\right\}_{i \in I} \subset X$ there exists $j \in I$ such that $x_{j} \in \operatorname{wcl}\left(\left\{x_{i}\right\}_{i \in I \backslash\{j\}}\right)$. Clearly, $\mathrm{KS}_{6} \Rightarrow \mathrm{KS}_{5}$. It seems that the only known example of a Banach space $X$ such that $X \in \mathrm{KS}_{6}$ is the space $X=C(K), K$ being the Kunen compact space ([8, p. 1123]) constructed by Kunen under $\mathrm{CH}$. This space $C(K)$ of Kunen has more interesting pathological properties. For example, $\left((C(K))^{n}, w^{n}\right)$ is hereditarily Lindelöf for every $n \in \mathbb{N}$.

In view of this situation, we can introduce the property $\mathrm{KS}_{7}$. A Banach space $X$ is said to have the Kunen-Shelah property $\mathrm{KS}_{7}$ if $\left(X^{n}, w^{n}\right)$ is hereditarily Lindelöf for every $n \in \mathbb{N}$. It can be easily proved that $\mathrm{KS}_{7} \Rightarrow \mathrm{KS}_{6}$. We know neither if the Shelah space $S$ has the property $\mathrm{KS}_{6}$ nor if the properties $\mathrm{KS}_{5}, \mathrm{KS}_{6}$ and $\mathrm{KS}_{7}$ are inequivalent.

\section{References}

[1] C. Finet and G. Godefroy, Biorthogonal systems and big quotient spaces, in: Contemp. Math. 85, Amer. Math. Soc., 1989, 87-110.

[2] A. S. Granero, Some uncountable structures and the Choquet-Edgar property in non-separable Banach spaces, in: Proc. 10th Spanish-Portuguese Conf. in Math. III, Murcia, 1985, 397-406.

[3] A. S. Granero, M. Jiménez Sevilla and J. P. Moreno, On $\omega$-independence and the Kunen-Shelah property, Proc. Edinburgh Math. Soc. 45 (2002), 391-395.

[4] M. Jiménez Sevilla and J. P. Moreno, Renorming Banach spaces with the Mazur intersection property, J. Funct. Anal. 144 (1997), 486-504.

[5] W. Johnson and J. Lindenstrauss, Some remarks on weakly compactly generated Banach spaces, Israel J. Math. 17 (1974), 219-230.

[6] N. J. Kalton, Independence in separable Banach spaces, in: Contemp. Math. 85, Amer. Math. Soc., 1989, 319-323.

[7] J. Lindenstrauss and L. Tzafriri, Classical Banach Spaces I, Springer, 1977.

[8] S. Negrepontis, Banach spaces and topology, in: Handbook of Set-Theoretic Topology, North-Holland, 1984, 1045-1142.

[9] A. N. Plichko, Some properties of the Johnson-Lindenstrauss space, Funct. Anal. Appl. 15 (1981), 88-89.

[10] R. Pol, On a question of H. H. Corson and some related problems, Fund. Math. 109 (1980), 143-154.

[11] W. Rudin, Real and Complex Analysis, McGraw-Hill, 1974. 
[12] A. Sersouri, $\omega$-independence in nonseparable Banach spaces, in: Contemp. Math. 85, Amer. Math. Soc., 1989, 509-512.

[13] S. Shelah, Uncountable constructions for B.A., e.c. groups and Banach spaces, Israel J. Math. 51 (1985), 273-297.

[14] I. Singer, Bases in Banach Spaces II, Springer, 1981.

[15] D. van Dulst, Reflexive and Superreflexive Banach Spaces, Math. Centrum, Amsterdam, 1978.

Departamento Análisis Matemático

Facultad de Matemáticas

Universidad Complutense

Madrid 28040, Spain

E-mail: granero@mat.ucm.es marjim@mat.ucm.es montesinos@mat.ucm.es

Departamento de Matemáticas

Facultad de Ciencias

Universidad Autónoma de Madrid

Madrid 28049, Spain

E-mail: josepedro.moreno@uam.es
Department of Mathematics Pedagogical University Shevchenko st. 1 Kirovograd 2506, Ukraine E-mail: aplichko@kspu.kr.ua

Received December 30, 2002 